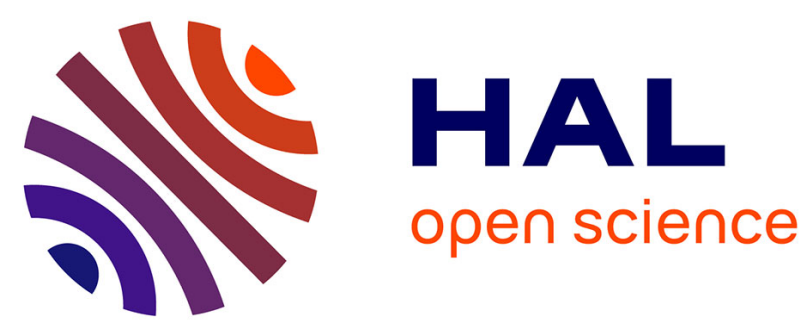

\title{
Hybrid micromechanical-phenomenological modelling of anisotropic damage and anelasticity induced by micro-cracks in unidirectional composites
}

Francis Praud, Georges Chatzigeorgiou, Yves Chemisky, Fodil Meraghni

\section{- To cite this version:}

Francis Praud, Georges Chatzigeorgiou, Yves Chemisky, Fodil Meraghni. Hybrid micromechanical-phenomenological modelling of anisotropic damage and anelasticity induced by micro-cracks in unidirectional composites. Composite Structures, 2017, 182, pp.223-236. 10.1016/j.compstruct.2017.09.013 . hal-01634479

\section{HAL Id: hal-01634479 \\ https://hal.science/hal-01634479}

Submitted on 22 Nov 2017

HAL is a multi-disciplinary open access archive for the deposit and dissemination of scientific research documents, whether they are published or not. The documents may come from teaching and research institutions in France or abroad, or from public or private research centers.
L'archive ouverte pluridisciplinaire HAL, est destinée au dépôt et à la diffusion de documents scientifiques de niveau recherche, publiés ou non, émanant des établissements d'enseignement et de recherche français ou étrangers, des laboratoires publics ou privés. 


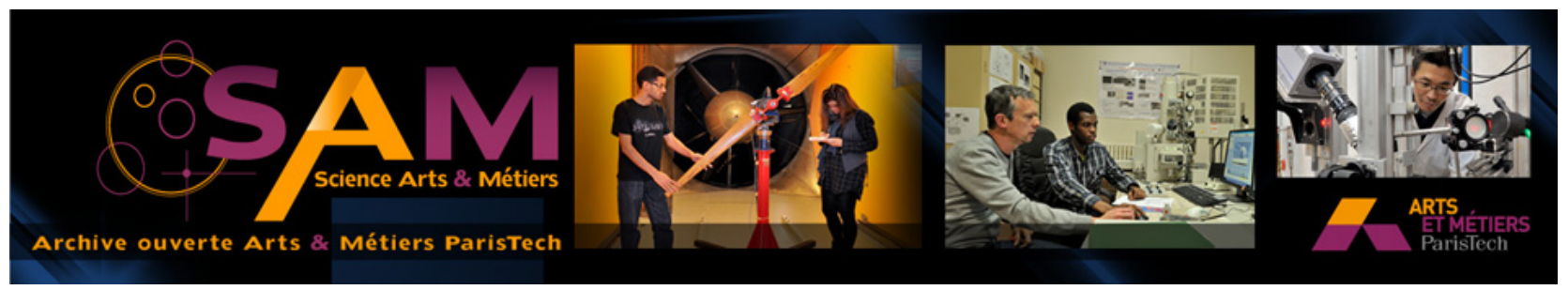

Science Arts \& Métiers (SAM)

is an open access repository that collects the work of Arts et Métiers ParisTech researchers and makes it freely available over the web where possible.

This is an author-deposited version published in: http://sam.ensam.eu

Handle ID: .http://hdl.handle.net/null

\section{To cite this version :}

Francis PRAUD, George CHATZIGEORGIOU, Yves CHEMISKY, Fodil MERAGHNI - Hybrid micromechanical-phenomenological modelling of anisotropic damage and anelasticity induced by micro-cracks in unidirectional composites - Composite Structures - Vol. 182, p.223-236 - 2017 


\title{
Hybrid micromechanical-phenomenological modelling of anisotropic damage and anelasticity induced by micro-cracks in unidirectional composites
}

\author{
F. Praud ${ }^{\mathrm{a}, \mathrm{b}}$, G. Chatzigeorgiou ${ }^{\mathrm{a}}$, Y. Chemisky ${ }^{\mathrm{a}}$, F. Meraghni ${ }^{\mathrm{a}, *}$ \\ ${ }^{a}$ Arts et Metiers ParisTech, LEM3-UMR 7239 CNRS, 4 rue Augustin Fresnel, 57078 \\ Metz, France \\ ${ }^{b}$ IRT Jules Verne, Chemin du Chaffault, 44340 Bougenais, France
}

\begin{abstract}
In this paper, a new modelling approach is presented to describe the damaged mechanical response of unidirectional composites under the small strain assumption. The proposed constitutive equations inherits from the phenomenological theories of Continuum Damage Mechanics (CDM) but brings out a micromechanical description of the damaged Representative Volume Element (RVE) while being formulated in a proper thermodynamical framework. The model is provided with an implicit numerical scheme based on the so-called "return mapping algorithm" as well as the formulation of the tangent operator. The identification and the prediction capabilities of the model are validated using experimental data including off-axis tensile tests. Finally, to provide a better understanding of the model, a multi-axial non-proportional simulation is performed and analysed.
\end{abstract}

Keywords: Non-linear behaviour; Anisotropic damage; Micromechanics;

${ }^{*}$ Corresponding author. Tel: $+33(0) 387375459$, Fax $:+33(0) 387374284$

Email address: fodil.meraghni@ensam.eu (F. Meraghni) 
Implicit implementation; Unidirectional composites. 


\section{Introduction}

The development of unidirectional composites has been driven by the necessity to develop lightweight new structures with enhanced mechanical properties, especially for automotive and transportation industries. The design of such structural components requires the deep understanding of their mechanical behaviour and failure mechanisms. In composite materials, the damage mechanisms are governed by the specific arrangement of the reinforcement, leading to an anisotropic evolution of their mechanical response. For the case of unidirectional composites reinforced with stiff fibres (e.g., glass or carbon), the longitudinal behaviour exhibits a linear elastic response until the material brittle failure due to fibres breakage. The presence of continuous fibrous reinforcements actually prevents the appearance of others damage mechanisms in the fibre direction. The transverse tension and the in-plane shear responses generally exhibit progressive stiffness degradation prior to failure. Indeed, transverse damage is induced by the appearance of a diffuse micro-crack network that initiates by debonding at the fibre/matrix interfaces (Figure 1a) and propagates by coalescence (Figure 1b). 


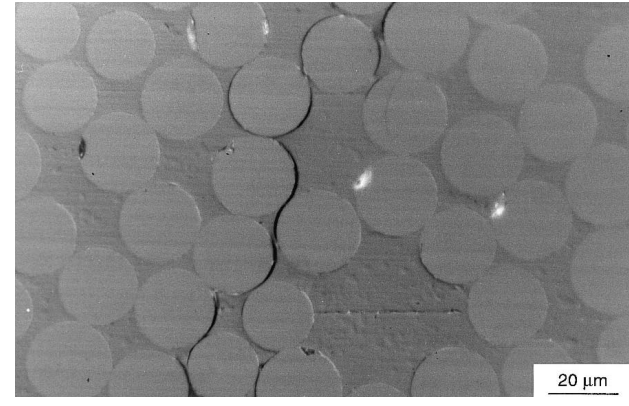

(a) Initiation of the micro-cracks by debonding of the fibre/matrix interfaces

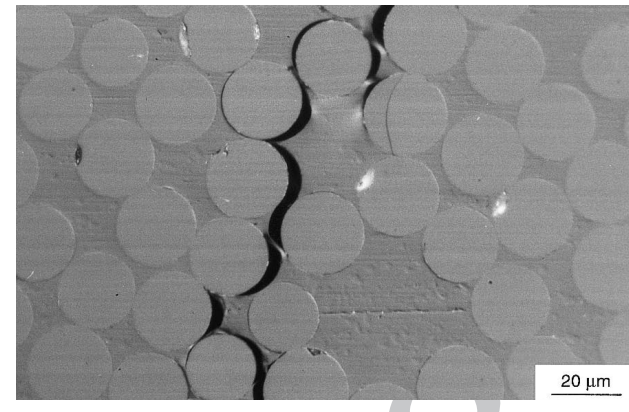

(b) Coalescence into micro-cracks

Figure 1: Transverse damage mechanism in unidirectional composites (Gamstedt and Sjögren, 1999).

Most of the modelling efforts have therefore focused on the definition of damage variables related to the previously described behaviour. Continuum Damage Mechanics (CDM) along with the concept of effective stress formulated within the framework of thermodynamics (Lemaitre and Chaboche, 1990; Lemaitre and Desmorat, 2005) is particularly adapted for such description. Initially developed in the context of isotropic material response, the CDM was adapted to the anisotropic case of unidirectional composites (Le Dantec, 1989; Ladevèze and Le Dantec, 1992; Ladevèze, 1992; Allix et al., 1994; Boubakar et al., 2002, 2003; Mahboob et al., 2017). The effects of micro-cracks are usually accounted through the introduction of several damage state variables that directly define the reduction of the material stiffness. In addition, the anelastic mechanisms are generally described by a plasticlike strain tensor whose evolution is governed by a yield function written in the effective stress space in order to account for the coupling with the damage. Nevertheless, if the use of CDM is convenient and straightforward in the 
isotropic case, its extension to anisotropy may lead to the following issues:

- Several damage state variables are assumed to capture properly the anisotropic evolution of the material response. Each variable is associated with an evolution equation that potentially depends on several field variables (temperature, stress) where multi-axial couplings may be included. Such a modelling strategy leads to an important number of material parameters with additional difficulties regarding their identification.

- The definition of the effective stress tensor is required to account for coupling effects with the anelastic mechanisms. In the case of anisotropic materials the physical meaning of the effective stress is not obvious (Lemaitre and Desmorat, 2005).

To overcome those issues, the evolution of the mechanical response of such composites can be rather based on the evolution of the micro-cracks network itself through micromechanics (Laws et al., 1983; Laws and Brockenbrough, 1987; Perreux and Oytana, 1993).

The main objective of this work is to propose a computationally efficient, hybrid micromechanical-phenomenological model that has a reduced number of internal variables. The evolution of these variables is controlled by criteria that depend on local stress estimations, providing hence a physically based description of the damage mechanisms. Such constitutive model is intended to be utilized for representing the response of bundles or yarns towards multiscale analyses of woven fabric or SMC composites. 
The new model accounts for anisotropic damage and anelasticity induced by micro-cracks in unidirectional composites. The damage is introduced through a micromechanical description of a Representative Volume Element (RVE) containing micro-cracks. Those defects are quantified by a unique internal state variable whose evolution is governed by a local stress criterion. The anisotropic evolution of the stiffness as well as the connection between overall and local stress-strain fields are therefore determined using micromechanical relationships that are directly incorporated within the constitutive equations. In that sense, the proposed model is based on a hybrid micromechanical-phenomenological formulation (Couégnat, 2008; Zhu et al., 2011; Qi et al., 2016). A novelty of the proposed model consists in accounting for anelastic deformation mechanisms by introducing the concept of damage induced anelasticity, where permanent strains are assumed to be caused by non-closure effect of the micro-cracks. The constitutive equations are then expressed within the framework of thermodynamics of irreversible processes applied to the overall medium, describing its mechanical response under the small strain assumption and isothermal conditions.

Compared to classical CDM models for unidirectional composites (Le Dantec, 1989; Ladevèze and Le Dantec, 1992; Ladevèze, 1992; Allix et al., 1994; Boubakar et al., 2002, 2003; Mahboob et al., 2017), the present modelling strategy leads to a reduced number of parameters with a certain ease regarding their identification. Moreover, the hybrid micromechanicalphenomenological formulation brings a physical basis to the model, while its 
practical use remains as computationally efficient as any purely phenomenological approach.

This paper is structured as follows: In the second section, the constitutive equations, the thermodynamical framework and the micro-mechanical aspects of the proposed model are presented. The third section is devoted to the numerical time implicit implementation scheme in which a user material algorithm is formulated alongside the definition of the tangent operator, essential for use in the finite element framework. The fourth section focuses on the identification strategy of the model parameters. The fifth section presents an example of simulation where the material is subjected to a non-proportional multi-axial loading. The last section summarizes the main conclusions and discuses the perspective related to this work.

The following notation is adopted in this work: bold and blackboard symbols respectively denote second and fourth order tensors while other symbols are scalar quantities. The twice contracted and dyadic products are given by:

$$
\boldsymbol{A}: \boldsymbol{B}=A_{i j} B_{i j}, \quad(\mathbb{A}: \boldsymbol{B})_{i j}=A_{i j k l} B_{k l}, \quad(\boldsymbol{A} \otimes \boldsymbol{B})_{i j k l}=A_{i j} B_{k l} .
$$

Moreover, all the second order tensors are symmetric $\left(A_{i j}=A_{j i}\right)$ and all the fourth order tensors have at least the minor symmetries $\left(A_{i j k l}=A_{j i k l}=\right.$ $\left.A_{i j l k}\right)$. Consequently, they can be respectively reduced to $6 \times 1$ and $6 \times 6$ matrices according to the Voigt notation. I and $\mathbb{I}$ are the second and the 
fourth order identity tensors, respectively, defined as:

$$
(\boldsymbol{I})_{i j}=\delta_{i j}, \quad(\mathbb{I})_{i j k l}=\frac{1}{2}\left(\delta_{i k} \delta_{j l}+\delta_{i l} \delta_{j k}\right),
$$

where $\delta_{i j}$ is the Kronecker symbol. The inverse of a fourth order tensor $\mathbb{A}$, that has the minor symmetries, is the fourth order tensor $\mathbb{A}^{-1}$ for which $\mathbb{A}: \mathbb{A}^{-1}=\mathbb{A}^{-1}: \mathbb{A}=\mathbb{I}$.

\section{Constitutive model and thermodynamical framework}

The new idea of the proposed model is to link, through micromechanical concepts, the overall stiffness reduction of a unidirectional composite with the evolution of a single scalar state variable $\gamma_{c}$ that quantifies the state of the involved damage mechanism, the oriented micro-cracking in the present case. This stiffness reduction is represented under the form of a fourth order tensor $\mathbb{D}\left(\gamma_{c}\right)$ that gradually decreases the initial stiffness tensor $\mathbb{C}_{\mathbf{0}}$ of the material, the latter having transversely isotropic properties as it represents the elastic behaviour of the undamaged unidirectional composite (initial material). The tensor $\mathbb{D}\left(\gamma_{c}\right)$ is assessed by homogenization of the initial material in which a micro-crack density $\gamma_{c}$ is introduced (Figure 2). The latter is defined as a void volume fraction created when the material is being damaged. Due to the micro-structure arrangement the micro-crack are forced to propagate in a plane parallel to the fibre direction $\vec{x}_{1}$. Moreover, if the material is mainly loaded in plane stress (plane $\vec{x}_{1}, \vec{x}_{2}$ ) as it is often the case, then the propagation plane of the micro-cracks can be considered as being perpendicular to the second direction $\vec{x}_{2}$ and consequently always oriented in the same plane (Figure 2). 
i)

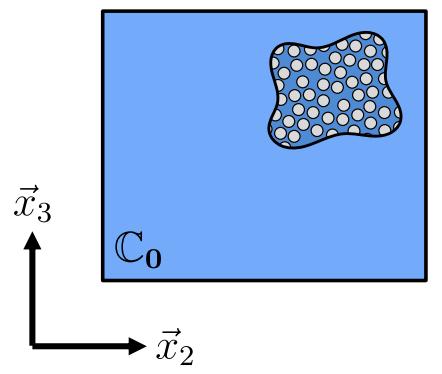

ii)

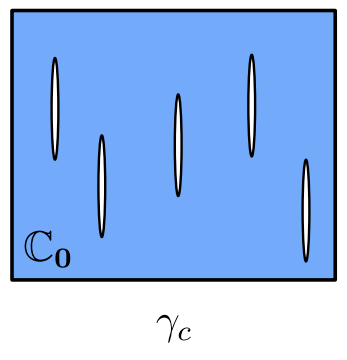

iii)

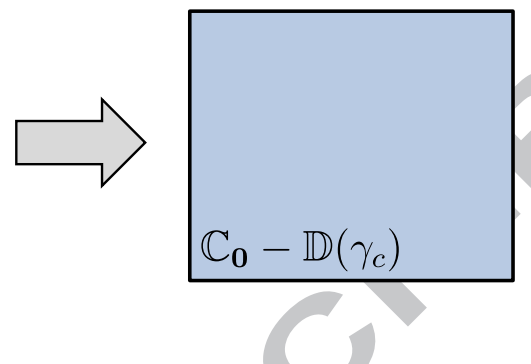

Figure 2: i) Initial state: transversely isotropic. ii) Damaged state: introduction of a micro-cracks density $\gamma_{c}$ (void volume fraction) in the RVE. iii) Evaluation of the stiffness reduction induced by $\gamma_{c}$ by homogenization.

Damage in such type of materials generally brings about permanent strains due to the micro-cracks non-closure and the resultant sliding with friction (Le Dantec, 1989; Ladevèze and Le Dantec, 1992; Boubakar et al., 2002, 2003; Mahboob et al., 2017). In the present model, these damage induced permanent strains are phenomenologically described by an anelastic strain tensor denoted by $\varepsilon_{s}$. The total overall strain is then expressed by summation of the elastic strain $\varepsilon_{e}$ with the anelastic strain $\varepsilon_{s}$ :

$$
\varepsilon=\varepsilon_{e}+\varepsilon_{s}
$$

The observable state variable of the model is the total strain $\varepsilon$ while the internal state variables are $\gamma_{c}$ and $\varepsilon_{s}$. The elastic strain $\varepsilon_{e}$ is not considered as a state variable but, for convenience, is expressed as the difference between the total strain and the anelastic strain.

\subsection{State laws}

According to the framework of thermodynamics (Lemaitre and Chaboche, 1990), the state laws are based on the postulate of a state potential. For the 
proposed model, the Helmholtz free energy is chosen to describe the energetic state of the material. The latter is formulated as a stored energy function of the state variables:

$$
\rho \psi\left(\varepsilon, \varepsilon_{s}, \gamma_{c}\right)=\frac{1}{2}\left(\varepsilon-\varepsilon_{s}\right):\left[\mathbb{C}_{0}-\mathbb{D}\left(\gamma_{c}\right)\right]:\left(\varepsilon-\varepsilon_{s}\right)
$$

The associated thermodynamic variables, namely the stress $\boldsymbol{\sigma}, \boldsymbol{- \sigma}$ and the energy density release $-Y_{c}$ are expressed by derivation of the potential with respect to their corresponding state variables $\varepsilon, \varepsilon_{s}$ and $\gamma_{c}$, respectively:

$$
\begin{gathered}
\boldsymbol{\sigma}=\rho \frac{\partial \psi}{\partial \boldsymbol{\varepsilon}}=\left[\mathbb{C}_{\mathbf{0}}-\mathbb{D}\left(\gamma_{c}\right)\right]:\left(\boldsymbol{\varepsilon}-\boldsymbol{\varepsilon}_{\boldsymbol{s}}\right), \\
-\boldsymbol{\sigma}=\rho \frac{\partial \psi}{\partial \boldsymbol{\varepsilon}_{\boldsymbol{s}}}, \\
-Y_{c}=\rho \frac{\partial \psi}{\partial \gamma_{c}}=-\frac{1}{2}\left(\boldsymbol{\varepsilon}-\boldsymbol{\varepsilon}_{\boldsymbol{s}}\right): \frac{\partial \mathbb{D}\left(\gamma_{c}\right)}{\partial \gamma_{c}}:\left(\boldsymbol{\varepsilon}-\boldsymbol{\varepsilon}_{\boldsymbol{s}}\right) .
\end{gathered}
$$

The state and associated variables of the proposed model are summarized in Table 1.

Table 1: State and associated variables.

\begin{tabular}{ccc}
\hline \multicolumn{2}{c}{ State variables } & Associated viariables \\
\hline Observable & internal & - \\
\hline$\varepsilon$ & & $\boldsymbol{\sigma}$ \\
& $\varepsilon_{s}$ & $-\boldsymbol{\sigma}$ \\
& $\gamma_{c}$ & $-Y_{c}$ \\
\hline
\end{tabular}




\subsection{Micro-mechanical aspects}

Obviously, the state laws formulated on the previous section depend on the exact physical signification given to the micro-crack density $\gamma_{c}$ along with the definition of the stiffness reduction tensor $\mathbb{D}\left(\gamma_{c}\right)$. As previously mentioned, in the proposed model, it has been chosen to identify these quantities using micro-mechanical concepts (Laws et al., 1983; Laws and Brockenbrough, 1987; Perreux and Oytana, 1993; Couégnat, 2008).

\subsubsection{Definition of the stiffness reduction}

By assuming micro-cracks as oriented quasi flat ellipsoidal inclusions of void, an explicit expression of the stiffness tensor $\mathbb{D}\left(\gamma_{c}\right)$ can be obtained according to the void volume fraction $\gamma_{c}$, also referred as the micro-crack density (Meraghni and Benzeggagh, 1995; Meraghni et al., 1996, 2002). The voids represents the micro-discontinuities as a part of the material that is unable to sustain stress. This is taken into account by considering zero stiffness tensor for the void inclusions. Using mean field approaches (Qu and Cherkaoui, 2006), the overall stiffness of the damaged composite, namely $\mathbb{C}_{\mathbf{0}}-\mathbb{D}\left(\gamma_{c}\right)$, is determined from a two phases RVE consisting of a volume fraction $\gamma_{c}$ of void inclusions (with zero stiffness) embedded in a reference medium (the virgin part of the material in the present case). This gives:

$$
\mathbb{C}_{0}-\mathbb{D}\left(\gamma_{c}\right)=\left(1-\gamma_{c}\right) \mathbb{C}_{0}: \mathbb{A}_{0}\left(\gamma_{c}\right)
$$

In the above equation, $\mathbb{A}_{\mathbf{0}}\left(\gamma_{c}\right)$ is the strain localisation tensor that is to be defined. The strain localisation in the void inclusions is represented by the tensor $\mathbb{A}_{\boldsymbol{c}}\left(\gamma_{c}\right)$. It is well established ( $\mathrm{Qu}$ and Cherkaoui, 2006) that, for a 
two phases RVE, one can write the following relationship between the strain localisation tensors:

$$
\left(1-\gamma_{c}\right) \mathbb{A}_{\mathbf{0}}\left(\gamma_{c}\right)+\gamma_{c} \mathbb{A}_{c}\left(\gamma_{c}\right)=\mathbb{I}
$$

According to the micromechanical scheme of Mori and Tanaka (1973), the strain localisation in the inclusions $\mathbb{A}_{\boldsymbol{c}}\left(\gamma_{c}\right)$ is calculated from the strain localisation in the reference medium $\mathbb{A}_{\mathbf{0}}\left(\gamma_{c}\right)$, the latter is then directly deduced from (7):

$$
\mathbb{A}_{\boldsymbol{c}}\left(\gamma_{c}\right)=\mathbb{T}_{\boldsymbol{c}}: \mathbb{A}_{\mathbf{0}}\left(\gamma_{c}\right) \quad \text { and } \quad \mathbb{A}_{\mathbf{0}}\left(\gamma_{c}\right)=\left(\mathbb{I}+\gamma_{c}\left(\mathbb{T}_{\boldsymbol{c}}-\mathbb{I}\right)\right)^{-1}
$$

where $\mathbb{T}_{\boldsymbol{c}}$ is the interaction tensor obtained from the solution of Eshelby (1957). For void inclusions with zero stiffness, this interaction tensor can be easily expressed as:

$$
\mathbb{T}_{c}=\left(\mathbb{I}-\mathbb{S}_{\boldsymbol{E}}\right)^{-1}
$$

$\mathbb{S}_{\boldsymbol{E}}$ is the well known Eshelby tensor (Eshelby, 1957) that depends on the stiffness tensor of the reference medium $\mathbb{C}_{0}$ and the relative dimensions $a_{1}, a_{2}$ and $a_{3}$ (Figure 3 ) of the void ellipsoidal inclusion. Because of the anisotropic properties of the reference medium $\left(\mathbb{C}_{\mathbf{0}}\right)$, the Eshelby tensor $\mathbb{S}_{\boldsymbol{E}}$ must be numerically evaluated using the methology proposed by Gavazzi and Lagoudas (1990) that is briefly presented in Appendix A. Finally, the introduction of (7) and (8) into (6) leads to the following formulation of the overall stiffness tensor of the damaged material, hightligting the expression of the stiffness reduction tensor $\mathbb{D}\left(\gamma_{c}\right)$ :

$$
\mathbb{C}_{0}-\mathbb{D}\left(\gamma_{c}\right)=\mathbb{C}_{0}-\gamma_{c} \mathbb{C}_{0}: \mathbb{T}_{c}: \mathbb{A}_{0}\left(\gamma_{c}\right)
$$


thus,

$$
\mathbb{D}\left(\gamma_{c}\right)=\gamma_{c} \mathbb{C}_{\mathbf{0}}: \mathbb{T}_{\boldsymbol{c}}: \mathbb{A}_{\mathbf{0}}\left(\gamma_{c}\right)
$$

Besides computing the stiffness reduction, the strain localisation tensors (8) allow to define the local strain fields, and more especially the one in the virgin part of the material:

$$
\varepsilon_{0}\left(\varepsilon, \varepsilon_{s}, \gamma_{c}\right)=\mathbb{A}_{0}\left(\gamma_{c}\right):\left(\varepsilon-\varepsilon_{s}\right)
$$

Similarly, the local stress in the virgin part of the material $\sigma_{0}=\mathbb{C}_{\mathbf{0}}: \varepsilon_{\mathbf{0}}$ can be written either in term of strain $\boldsymbol{\varepsilon}$, or in term of stress $\boldsymbol{\sigma}$, if (3) is substituted:

$$
\boldsymbol{\sigma}_{\mathbf{0}}\left(\varepsilon, \boldsymbol{\varepsilon}_{\boldsymbol{s}}, \gamma_{c}\right)=\mathbb{C}_{\mathbf{0}}: \mathbb{A}_{\mathbf{0}}\left(\gamma_{c}\right):\left(\varepsilon-\boldsymbol{\varepsilon}_{\boldsymbol{s}}\right) \quad \text { or } \quad \boldsymbol{\sigma}_{\mathbf{0}}\left(\boldsymbol{\sigma}, \gamma_{c}\right)=\mathbb{B}_{\mathbf{0}}\left(\gamma_{c}\right): \boldsymbol{\sigma}
$$

where it can be demonstrated that the stress localisation tensor $\mathbb{B}_{\mathbf{0}}\left(\gamma_{c}\right)$, after proper calculation, is simply given by:

$$
\mathbb{B}_{\mathbf{0}}\left(\gamma_{c}\right)=\mathbb{C}_{\mathbf{0}}: \mathbb{A}_{\mathbf{0}}\left(\gamma_{c}\right):\left[\mathbb{C}_{\mathbf{0}}-\mathbb{D}\left(\gamma_{c}\right)\right]^{-1}=\frac{\mathbb{I}}{1-\gamma_{c}}
$$

It is easy to verify that, using the equations (8) and (14), when the material is undamaged: $\mathbb{A}_{\mathbf{0}}\left(\gamma_{c}=0\right)=\mathbb{B}_{\mathbf{0}}\left(\gamma_{c}=0\right)=\mathbb{I}$, then $\boldsymbol{\varepsilon}_{\mathbf{0}}=\left(\boldsymbol{\varepsilon}-\boldsymbol{\varepsilon}_{\boldsymbol{s}}\right)$ and $\boldsymbol{\sigma}_{\mathbf{0}}=\boldsymbol{\sigma}$. 


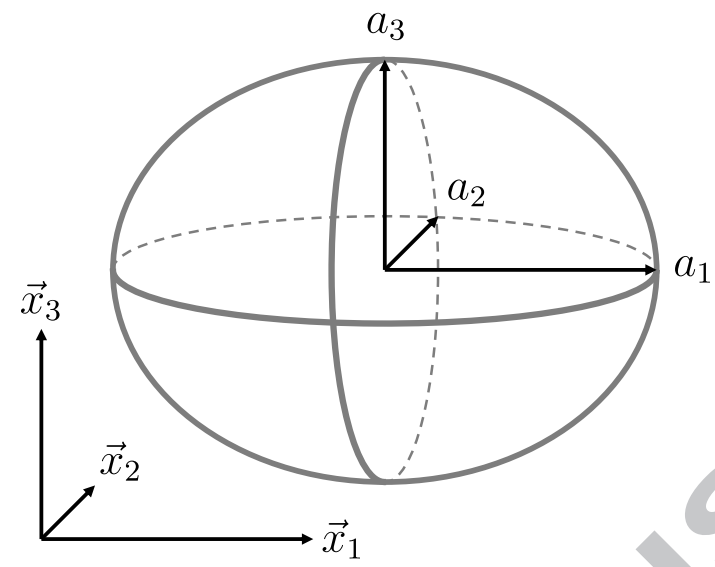

Figure 3: Ellipsoid with its relative dimensions.

Note that the interaction tensor $\mathbb{T}_{\boldsymbol{c}}$ is directly calculated from the Eshelby tensor $\mathbb{S}_{\boldsymbol{E}}$ in $(9)$ and consequently does not depends on any variables. Therefore, $\mathbb{T}_{\boldsymbol{c}}$ can be considered as an input of the model and is hence computed only one time prior any analysis. Such computational consideration is particularly important since the numerical evaluation of the Eshelby tensor is a computationally expensive task that must be avoided in a numerical process.

It is also important to point out that, although the tensors $\mathbb{S}_{\boldsymbol{E}}, \mathbb{T}_{\boldsymbol{c}}$ and $\mathbb{A}_{\mathbf{0}}$ do not have the major symmetry, while the tensor $\mathbb{D}\left(\gamma_{c}\right)$ always does. Indeed, for a two phases RVE (composite and void in the present case) the major symmetry of the homogenized stiffness tensor is ensured with the micromechanical scheme of Mori-Tanaka (Benveniste et al., 1991). This latter point is essential because it allows the definition of a thermodynamic state potential, as formulated in (2) for the proposed model. 
As mentioned in the introduction (Section 1), to consider anisotropic damage, CDM based model needs several damage state variables. With the proposed approach the definition of damage is provided through a micromechanical description. The anisotropic stiffness reduction induced by the micro-cracking is computed as a tensorial function which directly depends on the micro-crack density $\gamma_{c}$. The latter is denoted as an internal state variable, which has a physical signification from a micromechanical point of view.

\subsubsection{Geometrical aspects of the voids}

The orientation and the quasi flat aspect of the void ellipsoid inclusions imply specific conditions on their relative dimensions (Figure 3). Indeed, the flattening ratios $\delta_{1}$ and $\delta_{3}$ must tend to infinite values:

$$
\delta_{1}=\frac{a_{1}}{a_{2}} \rightarrow \infty, \quad \delta_{3}=\frac{a_{3}}{a_{2}} \rightarrow \infty .
$$

In practice, large enough value of flattening ratios are introduced for the numerical evaluation of the stiffness reduction. The latter is also influenced by the micro-cracks shape ratio $\delta_{c}$, where

$$
\delta_{c}=\frac{\delta_{1}}{\delta_{3}}=\frac{a_{1}}{a_{3}} .
$$

If $\delta_{c}=1$, then the shape of the micro-cracks is perfectly circular (penny shape). If $\delta_{c} \rightarrow \infty$, then the micro-cracks are crossing the volume element along $\vec{x}_{1}$, whereas in the opposite case, when $\delta_{c} \rightarrow 0$, the micro-cracks are crossing the volume element along $\vec{x}_{3}$. 
As an example, the stiffness reduction tensor $\mathbb{D}\left(\gamma_{c}\right)$ has been determined (Figure 4) for different configurations of void inclusions, as detailed in Table 2. For these computations, stiffness properties taken from a flax-epoxy unidirectional composite have been considered. These properties have been extracted from experimental data of Mahboob et al. (2017) as shown later in Section 4. The undamaged stiffness tensor of this material, namely $\mathbb{C}_{\mathbf{0}}$, is presented below with the help of the Voigt notation.

$$
\mathbb{C}_{\mathbf{0}}=\left(\begin{array}{ccccccc}
21975 & 2678 & 2678 & 0 & 0 & 0 \\
& 5711 & 1942 & 0 & 0 & 0 \\
& & 5711 & 0 & 0 & 0 \\
& & & & 2100 & 0 & 0 \\
& & & & 2100 & 0 \\
\text { sym. } & & & & & 1885
\end{array}\right) \quad \mathrm{MPa} .
$$

Note that $\mathbb{C}_{0}$ is well transversely isotropic as $C_{0_{2222}}=C_{0_{3333}}, C_{0_{1122}}=C_{0_{1133}}$, $C_{0_{1212}}=C_{0_{1313}}$ and $C_{0_{2323}}=\frac{1}{2}\left(C_{0_{2222}}-C_{0_{2233}}\right)=\frac{1}{2}\left(C_{0_{3333}}-C_{0_{2233}}\right)$. 
Table 2: Flattening and shape ratios of the void inclusions.

\begin{tabular}{lccc}
\hline configuration of the void inclusions & \multicolumn{2}{c}{ flattening ratios } & shape ratio \\
& $\delta_{1}=\frac{a_{1}}{a_{2}}$ & $\delta_{3}=\frac{a_{3}}{a_{2}}$ & $\delta_{c}=\frac{\delta_{1}}{\delta_{3}}=\frac{a_{1}}{a_{3}}$ \\
\hline \hline circular micro-cracks (penny shape) & 100 & 100 & 1 \\
circular micro-cracks (penny shape) & 200 & 200 & 1 \\
circular micro-cracks (penny shape) & 300 & 300 & 1 \\
circular micro-cracks (penny shape) & 400 & 400 & 1 \\
crossing micro-cracks along $\vec{x}_{1}$ & 400000 & 400 & 1000 \\
crossing micro-cracks along $\vec{x}_{3}$ & 400 & 400000 & 0.001 \\
\hline
\end{tabular}

It can be noticed that the presence of micro-cracks does not affect all the terms of the stiffness reduction tensor in the same way, leading to an anisotropic response in this damage mechanism. On Figure 4, it can be observed that the terms 1122, 2222, 2233, 1212 and 2323 of the stiffness are the most reduced, while the terms 1111, 3333 and 1313 are almost not affected for the considered range of micro-cracks density. The flattening of the voids inclusions appears to have a shift effect on the stiffness reduction. The influence of the flattening ratios $\delta_{1}$ and $\delta_{3}$ seems to become negligible from a certain value. Similar observations have been reported by Meraghni and Benzeggagh (1995) and validate the choice of large finite values to respect to respect infinite ratios. Regarding the shape of the cracks, it can be noticed that the micro-crack shape ratio $\delta_{c}$ mainly influences the stiffness reduction of the in-plane shear (term 1212), that appear to more important when microcracks cross the volume element along $\vec{x}_{1}$, namely when $\delta_{c} \rightarrow \infty$. 

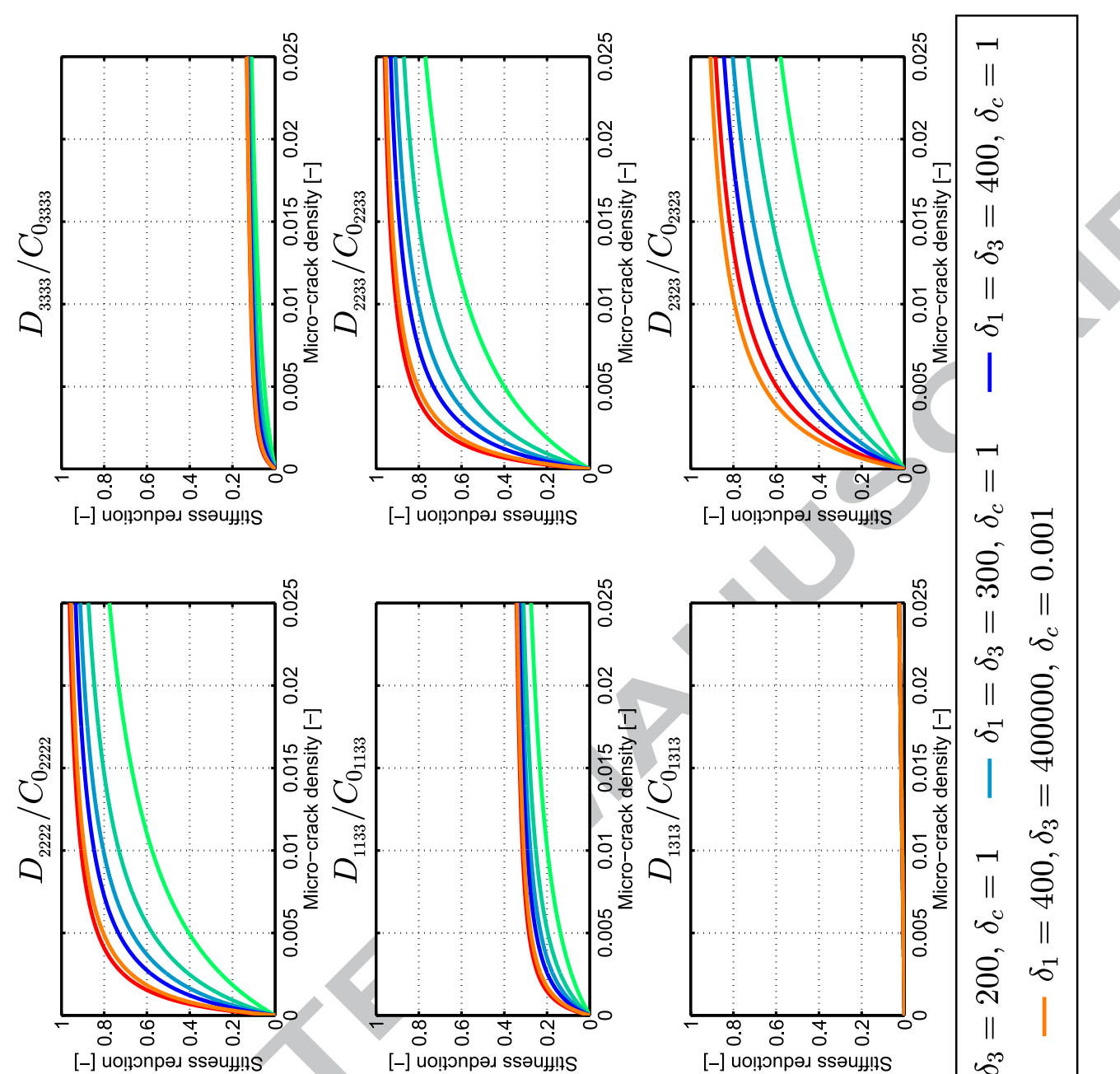

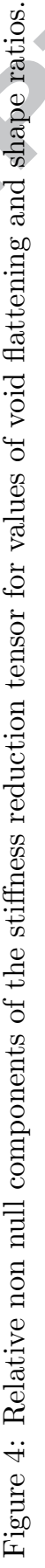




\subsection{Evolution laws}

Assuming no coupling between the thermal and mechanical dissipation, the second principle of thermodynamics implies that the mechanical dissipation has to be always positive or null through the Clausius Duhem inequality. The rate of the dissipated energy $\Phi$ is then expressed by the difference between the rate of the strain energy $W_{\varepsilon}$ and the rate of the stored energy $\rho \psi$, in which equations (3), (4) and (5) are substituted:

$$
\begin{aligned}
\dot{\Phi} & =\dot{W}_{\varepsilon}-\rho \dot{\psi} \geq 0 \\
& =\boldsymbol{\sigma}: \dot{\boldsymbol{\varepsilon}}-\rho\left(\frac{\partial \psi}{\partial \boldsymbol{\varepsilon}}: \dot{\boldsymbol{\varepsilon}}+\frac{\partial \psi}{\partial \varepsilon_{s}}: \dot{\boldsymbol{\varepsilon}}_{s}+\frac{\partial \psi}{\partial \gamma_{c}} \dot{\gamma}_{c}\right) \geq 0 \\
& =\boldsymbol{\sigma}: \dot{\boldsymbol{\varepsilon}}_{\boldsymbol{s}}+Y_{c} \dot{\gamma}_{c} \geq 0
\end{aligned}
$$

From an energetic point of view it is worth noticing that, according to the proposed model formulation, the internal energy function $\rho \psi$ presented in $(2)$ is only associated to the elastic part of the total strain. Indeed, in this formalism, there is no energetic term linked to the anelastic strain $\varepsilon_{s}$ that could permanently store energy in the material, like a hardening function in the case of plasticity. Consequently, $\rho \psi$ is fully and instantaneously recoverable when the stress is released. Thus, the non-linear mechanisms accounted in the model only dissipate and do not store energy in an unrecoverable manner (Figure 5). 


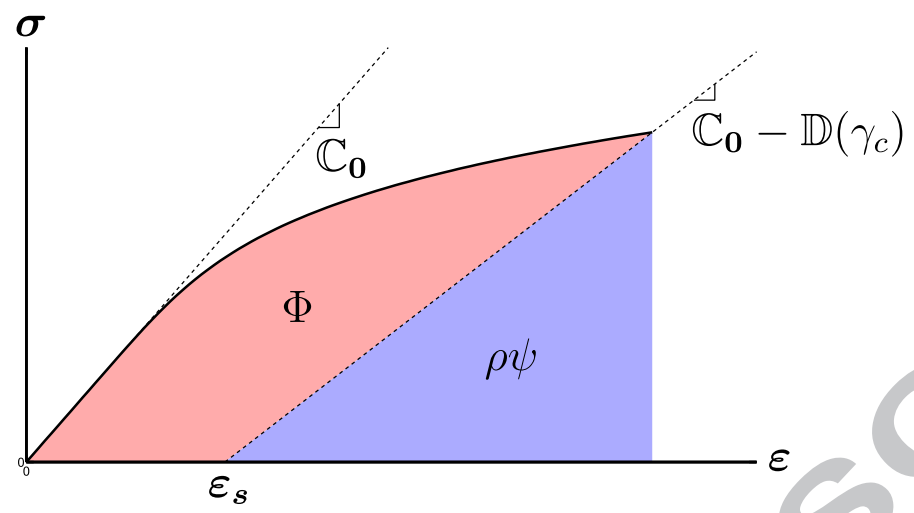

Figure 5: Balance between the dissipated $(\Phi)$ and stored $(\rho \psi)$ energies for the proposed model.

\subsubsection{Damage activation and development}

Classically, in most of the continuum damage models for unidirectional composite (Le Dantec, 1989; Ladevèze and Le Dantec, 1992; Ladevèze, 1992; Allix et al., 1994; Boubakar et al., 2002, 2003; Mahboob et al., 2017), the damage variables are driven by a criterion based on their associated variables, namely the energy density releases. In the present formulation, such a control would be inappropriate, since the growth of oriented micro-cracks is only generated under a certain type of stress state, namely when $\sigma_{22}$ and $\sigma_{12}$ are involved. For this reason, it is proposed to drive the development of the micro-crack density $\gamma_{c}$ by an anisotropic quadratic interaction criterion (Hill, 1948; Ottosen and Ristinmaa, 2005) expressed from the local stress in the virgin part of the material (13). This criterion, in its general form is given by:

$$
H_{c}\left(\boldsymbol{\sigma}_{\mathbf{0}}\right)=\sqrt{\boldsymbol{\sigma}_{\mathbf{0}}: \mathbb{H}: \boldsymbol{\sigma}_{\mathbf{0}}}
$$


In the above equation, $\mathbb{H}$ is a fourth order tensor configured in such a way that $H_{c}$ stays sensitive only to the components 22 and 12 of $\boldsymbol{\sigma}_{\mathbf{0}}$. With the help of the Voigt notation, this gives:

$$
H_{c}^{2}=\left\{\begin{array}{l}
\sigma_{0_{11}} \\
\sigma_{0_{22}} \\
\sigma_{0_{33}} \\
\sigma_{0_{12}} \\
\sigma_{0_{13}} \\
\sigma_{0_{23}}
\end{array}\right\} \times\left(\begin{array}{cccccc}
0 & 0 & 0 & 0 & 0 & 0 \\
& \frac{1}{R_{22}^{2}} & 0 & 0 & 0 & 0 \\
& & 0 & 0 & 0 & 0 \\
& & & \frac{1}{R_{12}^{2}} & 0 & 0 \\
& & & 0 & 0 \\
\text { sym. } & & & & 0
\end{array}\right\} \times\left\{\begin{array}{l}
\sigma_{0_{11}} \\
\sigma_{0_{22}} \\
\sigma_{0_{33}} \\
\sigma_{0_{12}} \\
\sigma_{0_{13}} \\
\sigma_{0_{23}}
\end{array}\right\}=\left(\frac{\sigma_{0_{22}}}{R_{22}}\right)^{2}+\left(\frac{\sigma_{0_{12}}}{R_{12}}\right)^{2} .
$$

As illustrated in Figure 6a, $R_{22}$ and $R_{12}$ denote the initial thresholds in pure transverse tension and in pure in-plane shear, respectively. According to the relationships (13) and (14), the local stress $\boldsymbol{\sigma}_{\mathbf{0}}$ and the overall stress $\boldsymbol{\sigma}$ have the same direction and a nearly equal amplitude since the micro-crack density $\gamma_{c}$ takes relatively small values. Nevertheless, a criterion based on this local stress $\boldsymbol{\sigma}_{\mathbf{0}}$ is more appropriate as it accounts for the neat part of the material sustaining the loading.

The development of the micro-crack density is formulated in such a way that the damage initiates only when $H_{c}$ exceeds 1 . After this stage, the micro-crack density $\gamma_{c}$ is expressed as a function $g$ of the maximal value reached by the criterion $H_{c}$ in the whole loading history, noted hereafter as $\sup \left(H_{c}\right)$. The function $g$ is chosen as a Weibull-like law (Weibull 1951) that is commonly utilized in micromechanics-based models to express the evolution of various types of damage mechanisms like micro-cracking (Derrien et al., 
2000; Meraghni et al., 2002) or interface debonding (Lee and Pyo, 2007; Zaïri et al., 2008; Despringre et al., 2016). Thus, in the proposed model, the development of the micro-crack density is expressed as follows:

$$
\gamma_{c}=g\left(\sup \left(H_{c}\right)\right)=\gamma_{c}^{\infty}\left[1-\exp \left(-\left[\frac{\left\langle\sup \left(H_{c}\right)-1\right\rangle_{+}}{S}\right]^{\beta}\right)\right],
$$

where $S$ and $\beta$ are the length and exponent parameters, respectively. $\gamma_{c}^{\infty}$ denotes the level of micro-crack saturation such that $\gamma_{c} \leq \gamma_{c}^{\infty}$, as shown in Figure 6b. If no saturation effect appears experimentally, then the parameter $\gamma_{c}^{\infty}$ can be simply fixed to a large enough value so that only the first part of the Weibull function $\left(0<\sup \left(H_{c}\right)<1+S\right)$ is acting (Figure $6 \mathrm{~b}$ ).

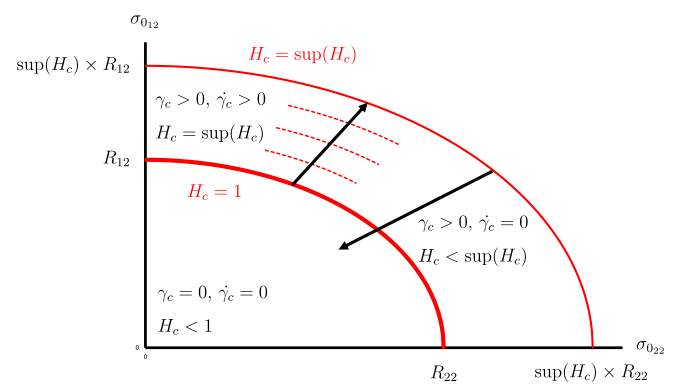

(a) Stress criterion $H_{c}$

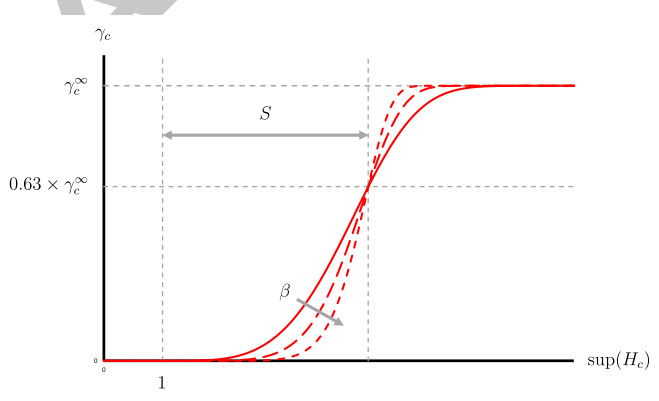

(b) Weibull-like law

Figure 6: Development of the micro-crack density.

It is worth noticing that the development relationship (21) accounts well for the irreversible aspect of damage implying that $\dot{\gamma}_{c} \geq 0$. Indeed, as illustrated in Figure 6a, damage is activated and evolves $\left(\dot{\gamma}_{c}>0\right)$ when $H_{c}=\sup \left(H_{c}\right)$, while it remains constant $\left(\dot{\gamma}_{c}=0\right)$ when $H_{c}<\sup \left(H_{c}\right)$. From a thermodynamic point of view, the inequality $Y_{c} \geq 0$ ensures that the dissipation related to the stiffness reduction is always positive or null, i.e. $Y_{c} \dot{\gamma}_{c} \geq 0$ 


\subsubsection{Evolution law for anelasticity}

Classically, in most of the CDM-based models for unidirectional composites (Le Dantec, 1989; Ladevèze and Le Dantec, 1992; Ladevèze, 1992; Allix et al., 1994; Boubakar et al., 2002, 2003; Mahboob et al., 2017), the anelastic mechanisms are formulated in a similar manner as plasticity, where the anelastic strains are governed by a yield criterion written in the effective stress space (Lemaitre and Chaboche, 1990; Lemaitre and Desmorat, 2005). Alternatively, the proposed approach considers that the anelasticity occurs as permanent strains caused by the micro-cracks non-closure upon unloading. Therefore, this anelasticity is a direct consequence of the micro-cracking that is represented by the internal state variable $\gamma_{c}$. Accordingly, the evolution of the anelastic strain $\varepsilon_{s}$ is directly linked to the variable of the micro-crack density $\gamma_{c}$, making the two mechanisms directly coupled and simultaneously activated. This evolution is expressed through the normality of a convex indicative function given by:

$$
F\left(\boldsymbol{\sigma}, Y_{c}\right)=H_{s}(\boldsymbol{\sigma})+Y_{c} \quad \text { with } \quad H_{s}(\boldsymbol{\sigma})=\sqrt{\boldsymbol{\sigma}: \mathbb{F}: \boldsymbol{\sigma}}
$$

In the above equation, $H_{s}$ is another anisotropic quadratic interaction criterion (Hill, 1948; Ottosen and Ristinmaa, 2005) in which the fourth order tensor $\mathbb{F}$ is configured in such a way that $H_{s}$ stays sensitive only to the 
components 22 and 12 of $\boldsymbol{\sigma}$. With the help of the Voigt notation, this gives:

$$
H_{s}^{2}=\left\{\begin{array}{l}
\sigma_{11} \\
\sigma_{22} \\
\sigma_{33} \\
\sigma_{12} \\
\sigma_{13} \\
\sigma_{23}
\end{array}\right\} \times\left(\begin{array}{cccccc}
0 & 0 & 0 & 0 & 0 & 0 \\
& a_{22}^{2} & 0 & 0 & 0 & 0 \\
& & 0 & 0 & 0 & 0 \\
& & & a_{12}^{2} & 0 & 0 \\
& & & 0 & 0 \\
\text { sym. } & & & & 0
\end{array}\right) \times\left\{\begin{array}{c}
\sigma_{11} \\
\sigma_{22} \\
\sigma_{33} \\
\sigma_{12} \\
\sigma_{13} \\
\sigma_{23}
\end{array}\right\},
$$

where $a_{22}$ and $a_{12}$ are material parameters. The normality of the indicative function $F\left(\boldsymbol{\sigma}, Y_{c}\right)$ allows to express the rate of $\gamma_{c}$ and $\varepsilon_{s}$ through a common multiplier $\lambda(\dot{\lambda} \geq 0)$ :

$$
\begin{gathered}
\dot{\gamma}_{c}=\frac{\partial F}{\partial Y_{c}} \dot{\lambda}=\dot{\lambda} \\
\dot{\boldsymbol{\varepsilon}}_{\boldsymbol{s}}=\frac{\partial F}{\partial \boldsymbol{\sigma}} \dot{\lambda}=\frac{\mathbb{F}: \boldsymbol{\sigma}}{H_{s}(\boldsymbol{\sigma})} \dot{\lambda} .
\end{gathered}
$$

The evolution of the multiplier, that appears to be the rate of the micro-crack density itself $\left(\dot{\gamma}_{c}=\dot{\lambda} \geq 0\right)$, is obtained through the damage development relationship (21). Then, by introducing (24) into (25) the evolution of the anelastic strain $\varepsilon_{\boldsymbol{s}}$ eventually takes the form of a flow equation:

$$
\dot{\varepsilon}_{s}=\Lambda_{s}(\boldsymbol{\sigma}) \dot{\gamma}_{c} \quad \text { with } \quad \boldsymbol{\Lambda}_{s}(\boldsymbol{\sigma})=\frac{\mathbb{F}: \boldsymbol{\sigma}}{H_{s}(\boldsymbol{\sigma})}
$$

In the above equation, $H_{s}(\boldsymbol{\sigma})$ is related to the direction of the anelastic strain, whereas $H_{c}\left(\boldsymbol{\sigma}_{\mathbf{0}}\right)$ is related to the damage activation and development as expressed in (21). In that sense the anelastic strain is governed by a nonassociative flow rule. The form of $\mathbb{F}$ in (23) implies that only the components 22 and 12 of the anelastic strain tensor $\varepsilon_{\boldsymbol{s}}$ are active and only evolve with the micro-crack density, namely when $\dot{\gamma}_{c}>0$. In addition, it is worth noting that 
the anelastic mechanism can be disactivated by setting $a_{22}=0$ and $a_{12}=0$.

\subsection{Remarks and discussion}

With the present model, the damage development and the direction of the anelastic strain are governed by the stress criteria $H_{c}(20)$ and $H_{s}(23)$, respectively. Although simple, the choices of these criteria are motivated by the fact that each one involves only two parameters that can be easily identified (see Section 4). It is worth recalling that the criteria Hc and Hs are both formulated as anisotropic quadratic interaction criteria based on the work of Hill (1948) and Ottosen and Ristinmaa (2005). These criteria imply symmetric envelopes in the stress space. Consequently, they cannot account for asymmetric effects such as the difference in response between tension and compression. Nevertheless, $H_{c}$ and $H_{s}$ can be easily replaced by other criteria (Hoffman, 1967; Tsai and Wu, 1971; Hashin and Rotem, 1973; Hashin, 1980; Karafillis and Boyce, 1993) accounting for more complicated multi-axial coupling and asymmetric effects. Such advanced criteria though would require additional parameters that need to be identified from experiments including tension-compression, and combined loading conditions. In the absence of such data, the proposed quadratic interaction criteria remain the best compromise.

As mentioned at the beginning of Section 2, the present model considers that the micro-cracks are always oriented in the same direction. This orientation is conditioned by the reinforcement and by an assumed plane stress state. In certain cases, this hypothesis could be violated. Indeed, according 
to the well-established Puck criterion (Puck and Schneider, 1969; Puck and Schürmann, 1998), it is known that the cracking plane may exhibit different types of orientation, especially when a transverse compressive stress is involved $\left(\sigma_{22}<0\right)$. The present modelling approach can be combined with such criterion to account for a load dependent micro-cracks orientation. However, integrating this feature may lead to several modelling difficulties. First, when the material is subjected to highly non-proportional loading, different cracking planes exist and may interact each other. Second, even though advanced algorithms have been already developed for the determination of a potential cracking plane (Wiegand et al., 2008; Schirmaier et al., 2014), the computational cost associated to this task is often prohibitive and the application of the algorithms in non-linear FE schemes is not straightforward.

Additionally, the introduction of local damage in FE structural analyses may cause additional difficulties, related to the localization effect. Indeed, this well-known unstable phenomenon caused by the loss of ellipticity of the system of equations cannot be described correctly with phenomenological models using local variables. Usually, in FE computations the localization is activated under any small perturbation, which may be caused by defects in the material, irregularities in the geometry of the structure or non-homogeneous boundary conditions. The numerical solution is then mesh dependent and the actual results are not reliable. Often, size effects and localization mechanisms can be captured with non-local models (Dimitrijevic and Hackl, 2008; Waffenschmidt et al., 2014; He et al., 2015; Kiefer et al., 2017), which use gradients of the variables and introduce a character- 
istic length. In the current work the proposed damage model is formulated and implemented in the framework of CDM with the assumption of diffuse damage prior to damage localization. All the numerical calculations are performed well before any unstable phenomenon occurs, thus the analyses do not suffer by loss of uniqueness. For structural applications the model can be considered accurate as long as the material at every point of the structure does not reach the limit where localization appears.

\section{Numerical implementation: Backward Euler time implicit algo- rithm}

When using a backward Euler time implicit numerical scheme, the value of a given quantity $x$ is computed from the previous time increment $n$ to the current one $n+1$ such that $x^{(n+1)}=x^{(n)}+\Delta x^{(n+1)}$. The latter relationship is implicit and usually requires an iterative scheme to be solved. Thus, the current value of the quantity $x$ is corrected for each iteration $k$ by: $x^{(n+1)(k+1)}=x^{(n+1)(k)}+\delta x^{(n+1)(k)}$ or $\Delta x^{(n+1)(k+1)}=\Delta x^{(n+1)(k)}+\delta x^{(n+1)(k)}$ until $x^{(n+1)}$ converges.

The implementation of a constitutive law in a finite element software like ABAQUS is usually ensured by a User MATarial subroutine (UMAT). When the analysis is completed at the time increment $n$, the finite element solver provides, for each integration point, all the state variables at the time increment $n$ along with the increment of total strain $\Delta \varepsilon^{(n+1)}$. Thus, from these data, the role of the UMAT subroutine is to compute: i) the stress and the 
state variables at the time increment $n+1$, and ii) the tangent operator $\mathbb{C}_{\boldsymbol{t}}$ that is necessary to achieve a fast convergence for the next finite element calculation.

The proposed scheme is based on the "convex cutting plane" form of the "return mapping algorithm" (Ortiz and Simo, 1986; Simo and Hughes, 1998). In such algorithms, it is convenient to write the evolution equations under the form of residual functions that must satisfy a nullity condition. With the proposed model, the damage development relationship (21) is written under the form of scalar function $\phi_{\gamma_{c}}$ that takes negative values when damage is not active $\left(\dot{\gamma}_{c}=0\right)$, or that must remain null when damage is active $\left(\dot{\gamma}_{c}>0\right)$ :

$$
\phi_{\gamma_{c}}\left(H_{c}, \gamma_{c}\right)=g\left(H_{c}\right)-\gamma_{c} \leq 0,\left\{\begin{array}{cc}
\text { if } \quad \phi_{\gamma_{c}}<0, & \dot{\gamma}_{c}=0 \\
\text { if } \quad \phi_{\gamma_{c}}=0, & \dot{\gamma}_{c}>0
\end{array} .\right.
$$

Note that the "convex cutting plane" method does not require the integration of the flow equation (26) as a residual function.

\subsection{Computation of the stress}

\subsubsection{Stress prediction}

In order to initiate the computation of the stress, the internal state variables are assumed not to evolve at first $\left(\Delta \gamma_{c}^{(n+1)(k=0)}=0\right.$ and $\Delta \varepsilon_{s}^{(n+1)(k=0)}=$ 0), while total strain at the end of the increment is given by $\varepsilon^{(n+1)}=$ $\varepsilon^{(n)}+\Delta \varepsilon^{(n+1)}$. Therefore, the stress is predicted and the residual $\phi_{\gamma_{c}}$ is then checked to identify whether the damage is active or not:

- If $\phi_{\gamma_{c}}^{(n+1)(k=0)} \leq 0$, then the damage is not active and consequently, the stress does not need to be corrected. 
- If $\phi_{\gamma_{c}}^{(n+1)(k=0)}>0$, then the damage is active. Thus, the "stress correction" is required.

\subsubsection{Stress correction}

During the "stress correction", the residual $\phi_{\gamma_{c}}$ returns back to zero by developing the internal state variables. It is reminded that, during this step, the total strain does not evolve: $\delta \boldsymbol{\varepsilon}^{(n+1)(k)}=\mathbf{0}$. Accordingly, the internal state variables are updated are updated at each iteration $k$ by:

$$
\gamma_{c}^{(n+1)(k+1)}=\gamma_{c}^{(n+1)(k)}+\delta \gamma_{c}^{(n+1)(k)}, \quad \varepsilon_{s}^{(n+1)(k+1)}=\boldsymbol{\varepsilon}_{s}^{(n+1)(k)}+\delta \varepsilon_{s}^{(n+1)(k)},
$$

where $\delta \gamma_{c}^{(n+1)(k)}$ and $\delta \varepsilon_{s}^{(n+1)(k)}$ are expressed through the linearisation of the constitutive equations (see Appendix B) along with the nullity condition of the residual $\phi_{\gamma_{c}}$ (see Appendix C). This eventually gives:

$$
\begin{gathered}
\delta \gamma_{c}^{(n+1)(k)}=\frac{-\phi_{\gamma_{c}}^{(n+1)(k)}}{K_{\gamma_{c} \gamma_{c}}^{(n+1)(k)}}, \\
\delta \boldsymbol{\varepsilon}_{\boldsymbol{s}}^{(n+1)(k)}=\boldsymbol{\Lambda}_{\boldsymbol{s}}\left(\boldsymbol{\sigma}^{(n+1)(k)}\right) \delta \gamma_{c}^{(n+1)(k)},
\end{gathered}
$$

where the expression of the "corrector" $K_{\gamma_{c} \gamma_{c}}$ is provided in (C.4). This correction procedure is iteratively repeated ( $k$ loop) until the convergence is reached, namely when $\left|\phi_{\gamma_{c}}^{(n+1)(k+1)}\right| \leq 0+\delta$.

Note that, according to the "convex cutting plane" method (Ortiz and Simo, 1986; Simo and Hughes, 1998), the flow equation (26) is explicitly integrated within the correction procedure. This is why, in (30), the flow $\boldsymbol{\Lambda}_{\boldsymbol{s}}$ is calculated from the previously updated stress $\boldsymbol{\sigma}^{(n+1)(k)}$. Nevertheless, it is worth pointing out that the time integration remains implicit. Despite this 
simplification, the "convex cutting plane" method provides a good accuracy compared to other schemes, while involving less computational cost (Qidwai and Lagoudas, 2000).

\subsection{Tangent operator}

In accordance with the "convex cutting plane" method (Ortiz and Simo, 1986; Simo and Hughes, 1998), the formulation of the tangent operator $\mathbb{C}_{\boldsymbol{t}}$ is obtained through the identification of a linear relationship between $d \boldsymbol{\sigma}$ and $d \varepsilon$ using a continuum description. When damage is not active $\left(\dot{\gamma}_{c}=0\right)$, the tangent operator is simply given by the current elastic stiffness: $\mathbb{C}_{\mathbf{0}}-\mathbb{D}\left(\gamma_{c}\right)$. When damage is active $\left(\dot{\gamma}_{c}>0\right)$, the stress-strain relationship is written in its linearised form. After proper calculation (see Appendix B), this gives:

$$
d \boldsymbol{\sigma}=\mathbb{B}_{\boldsymbol{\sigma} \varepsilon}: d \varepsilon+\boldsymbol{B}_{\boldsymbol{\sigma \gamma _ { c }}} d \gamma_{c}
$$

As damage is being generated, the condition $d \phi_{\gamma_{c}}=0$ must be satisfied. From the latter a linear relationship can be identified between $d \gamma_{c}$ and $d \varepsilon$ (see Appendix D):

$$
d \gamma_{c}=\boldsymbol{X}_{\gamma_{c} \varepsilon}: d \varepsilon
$$

where the term $\boldsymbol{X}_{\gamma_{c} \varepsilon}$ is the "tangent multiplier" whose expression is provided in (D.3). Finally, the substitution of (32) into (31) leads to the identification of the tangent operator $\mathbb{C}_{t}$, such that $d \boldsymbol{\sigma}=\mathbb{C}_{t}: d \varepsilon$. Its formulation is then given by:

$$
\mathbb{C}_{t}=\mathbb{B}_{\sigma \varepsilon}+\boldsymbol{B}_{\sigma \gamma_{c}} \otimes \boldsymbol{X}_{\gamma_{c} \varepsilon}
$$




\section{Model identification and experimental validation}

In this section, a suitable procedure is proposed for the identification of the model. This procedure is illustrated with experimental data coming from the work of Mahboob et al. (2017) on a flax-epoxy composite. Note that, compared to classical unidirectional composites with stiff fibres (e.g., glass or carbon), the case of the flax-epoxy is unusual. Indeed, due to the nature of the flax fibres, a slightly non-linear response can be observed in the longitudinal direction. It is important to mention that this peculiar aspect of the flax-epoxy composite cannot be properly captured with the proposed model. The latter has been preferentially developed for classical unidirectional composites and therefore assumes a linear response in the longitudinal direction. However, the transverse and in-plane shear behaviour in the flaxepoxy composite involves the same mechanisms than those described by the model. Consequently, in this study, the attention is more focused on the description of the transverse and in-plane shear, while in the longitudinal direction, the response is considered as being linear elastic with an average slope (see Figure 10a).

All the experimental data come from uni-axial tests performed on symmetric and balanced laminated specimens (Figure 7a). In these conditions, neither tension-shear nor tension-bending couplings appear, thus the overall laminate exhibits a purely uni-axial stress state in the specimen's coordinate system $(\vec{x}, \vec{y}, \vec{z})$. However, each single layer (Figure $7 \mathrm{~b})$, due to its orientation, is subjected to complex plane stress states in its local coordinate system $\left(\vec{x}_{1}, \vec{x}_{2}, \vec{x}_{3}\right)$. During the test a longitudinal strain $\varepsilon_{x x}$ was applied on the spec- 
imens, while the response in term of transversal strain $\varepsilon_{y y}$ and longitudinal stress were monitored $\sigma_{x x}$. Based on the individual behaviour of each single layer, the non-linear response of such multi-layered composite is simulated through a homogenisation scheme. Although the non-linear extension of the classical laminate theory is commonly used for this purpose (Berthelot, 1999; Perreux and Lazuardi, 2001), it is convenient to consider instead a scheme based on periodic homogenization (Chatzigeorgiou et al., 2015) in order to keep the constitutive model in its 3D formalism. Both methods actually provide the same results as long as laminates with a symmetric stacking are considered.

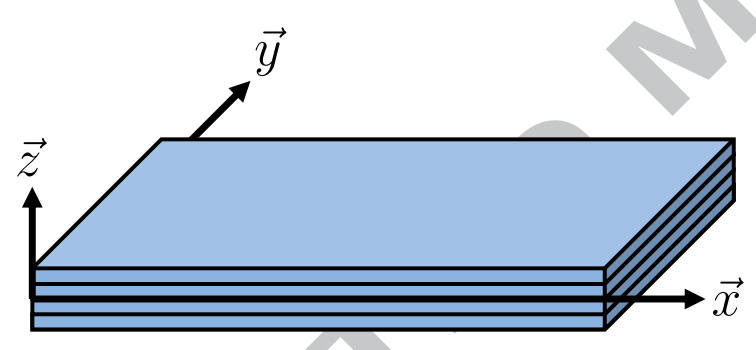

(a) Laminate: multi-layered composite

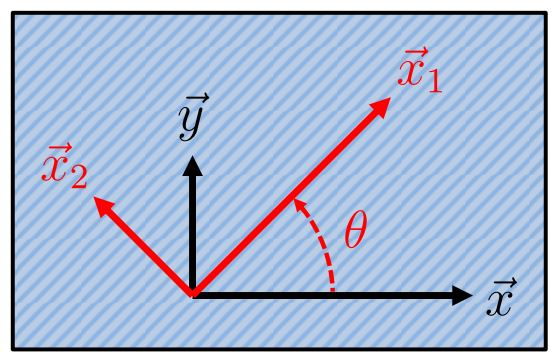

(b) Single layer

Figure 7: Definition of a laminate, $(\vec{x}, \vec{y}, \vec{z})$ is the coordinate system of the laminate, while $\left(\vec{x}_{1}, \vec{x}_{2}, \vec{x}_{3}\right)$ is the material one within a single layer.

It is proposed to identify, at first, the parameters related to the microcracks density evolution (21) and the anelasticity (26) in the transverse tension, namely, $R_{22}, S, \beta, \gamma_{c}^{\infty}$ and $a_{22}$. These parameters can be assessed through an incremental load/unload tensile test performed on a $\left[90^{\circ}\right]_{16}$ laminated specimen (Figure 8a). Indeed, with this stacking sequence the material, locally, is only subjected to a uni-axial stress state in the transverse direc- 
tion $\left(\sigma_{22}=\sigma_{x x}\right)$, thus the expression of the stress (3) can be reduced in its transverse uni-axial form. This gives:

$$
\sigma_{22}=\left[E_{0_{2}}-D_{2}\left(\gamma_{c}\right)\right]\left(\varepsilon_{22}-\varepsilon_{s_{22}}\right)
$$

where $E_{0_{2}}$ is the initial transverse Young modulus, while $D_{2}\left(\gamma_{c}\right)$ represents the transverse uni-axial stiffness reduction. It is important to mention that $D_{2}\left(\gamma_{c}\right)$ is not a term of the stiffness reduction tensor $\mathbb{D}\left(\gamma_{c}\right)$ but it is related to the latter by the following relationship:

$$
D_{2}\left(\gamma_{c}\right)=E_{0_{2}}-\frac{1}{S_{2222}\left(\gamma_{c}\right)} \quad \text { with } \quad \mathbb{S}\left(\gamma_{c}\right)=\left[\mathbb{C}_{\mathbf{0}}-\mathbb{D}\left(\gamma_{c}\right)\right]^{-1}
$$

When performing the test, at each stress level, the load release allows a direct measurement of the stiffness reduction $D_{2}$ as well as the anelastic strain $\varepsilon_{s_{22}}$, as illustrated in Figure 8a. Subsequently, considering the relationship (35), the micro-crack density corresponding to each stiffness reduction measurement is recovered (see Figure 8b). Once the damage threshold $R_{22}$ is identified (Figure 8a), it becomes easy to compute the stress criterion $H_{c}$ from (20) and (13). For a uni-axial positive stress state in the transverse direction, $H_{c}$ is reduced to:

$$
H_{c}=\frac{\sigma_{22}}{\left(1-\gamma_{c}\right) R_{22}}
$$

Then, the parameters $S, \beta, \gamma_{c}^{\infty}$ of the function $g(21)$, that expresses the relationship between the micro-crack density $\gamma_{c}$ and the criterion $H_{c}$, can be easily identified from the obtained experimental points (Figure 9a). Note that, for the considered material, it is observed that the saturation of damage is not reached at the end of the experiments (Figure 9a). Thus, the parameter $\gamma_{c}^{\infty}$, corresponding to the saturation regime, is set to a large enough value 
(see Table 3) so that only the first part of the function $g$ (21) is acting, as explained in Section 2.3.1. Still considering a uni-axial stress state in the transverse direction, the evolution law governing the anelastic strain (26) can be reduced to:

$$
\varepsilon_{s_{22}}=a_{22} \gamma_{c}
$$

Then, in the same way, the parameter $a_{22}$ is identified from the experimental points (Figure 9b).

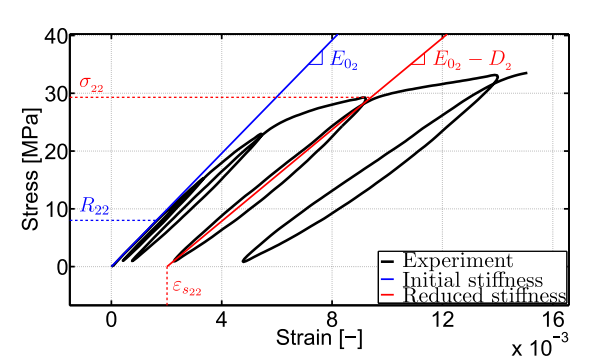

(a) Stress $\left(\sigma_{x x}\right)$ vs. strain $\left(\varepsilon_{x x}\right)$ curve performed on a $\left[90^{\circ}\right]_{16}$ laminate

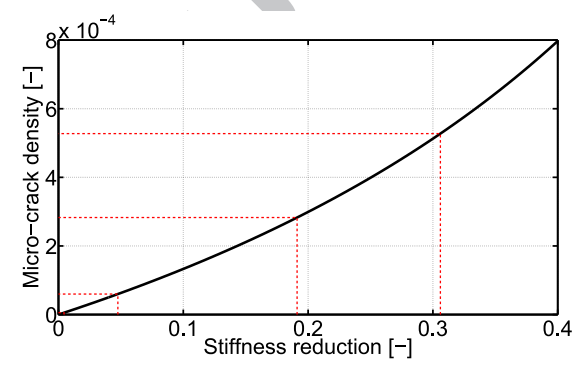

(b) Micro-crack density $\left(\gamma_{c}\right)$ vs. relative stiffness reduction $\left(D_{2} / E_{0_{2}}\right)$

Figure 8: For each stress level $\left(\sigma_{22}=\sigma_{x x}\right)$, the stiffness reduction $\left(D_{2}\right)$ as well as the anelastic strain $\left(\varepsilon_{s_{22}}\right)$ are measured. Thereafter, from (35), the micro-crack density $\left(\gamma_{c}\right)$ corresponding to each measurement of stiffness reduction is recovered. 


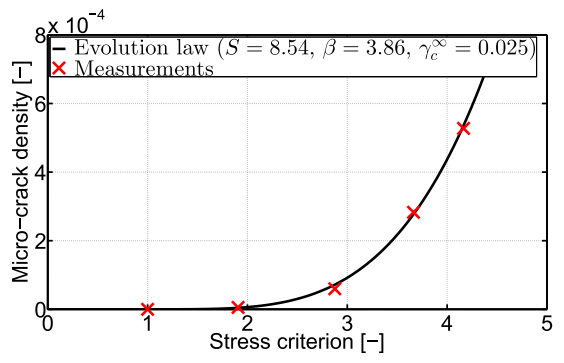

(a) Micro-crack density $\left(\gamma_{c}\right)$ vs. stress criterion $\left(H_{c}\right)$

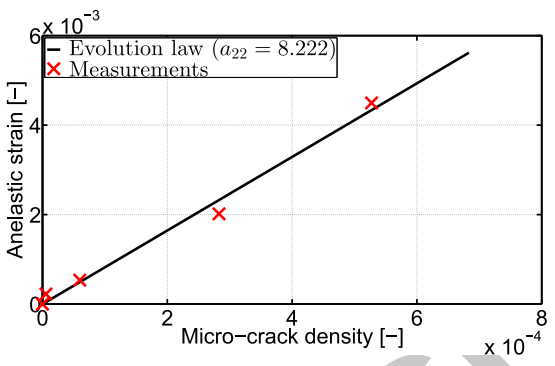

(b) Anelastic strain $\left(\varepsilon_{s_{22}}\right)$ vs. micro-crack density $\left(\gamma_{c}\right)$

Figure 9: Identification of the parameters related to the micro-crack density evolution and the anelasticity in the transverse direction, namely $S, \beta, \gamma_{c}^{\infty}$ and $a_{22}$.

Similarly to the previously described procedure, the remaining parameters, namely $R_{12}$ and $a_{12}$, could be determined from an in-plane shear test. Since pure shear tests are not easy to perform, it has been chosen to identify these parameters by mean of reverse engineering, from a load/unload tensile test performed on a $\left[ \pm 45^{\circ}\right]_{4 s}$ laminated specimen, where the damage is mainly governed by shear stress fields. Then the two remaining parameters are obtained by minimizing a cost function formulated from the least squares between the numerical and experimental mechanical response of the specimen (Meraghni et al., 2011, 2014). The obtained values of the parameters are listed in Table 3. 
Table 3: Identified parameters for flax-epoxy unidirectional composite.

\begin{tabular}{lccc}
\hline Feature & Parameter & value & unit \\
\hline \hline Pure transverse tension threshold & $R_{22}$ & 8 & $\mathrm{MPa}$ \\
Pure in-plane shear threshold & $R_{12}$ & 6 & $\mathrm{MPa}$ \\
Weibull length parameter & $S$ & 8.54 & - \\
Weibull exponent parameter & $\beta$ & 3.86 & - \\
Micro-cracks saturation (fixed) & $\gamma_{c}^{\infty}$ & 0.025 & - \\
Transverse tension anelasticity parameter & $a_{22}$ & 8.222 & - \\
In-plane shear anelasticity parameter & $a_{12}$ & 3.054 & - \\
\hline
\end{tabular}

The initial stiffness properties are provided in (17). They have been evaluated by measuring the initial slopes of the tensile tests performed on $\left[0^{\circ}\right]_{16}$, $\left[90^{\circ}\right]_{16}$ and $\left[ \pm 45^{\circ}\right]_{4 s}$ laminated specimens. It is worth recalling that, as explained in Section 2.2.1, the initial stiffness tensor $\mathbb{C}_{0}$ is required to compute the stiffness reduction along with a chosen configuration of void inclusions, in the present case : "crossing micro-cracks along $\vec{x}_{1}$ " (see Table 2).

Figures 10a, 10b and 10c show the comparison between the simulated and experimental data used for the identification, respectively, the tensile tests performed on $\left[0^{\circ}\right]_{16},\left[90^{\circ}\right]_{16}$ and $\left[ \pm 45^{\circ}\right]_{4 s}$ laminates. Despite a slight deviation on the transverse response on the $\left[ \pm 45^{\circ}\right]_{4 s}$ laminate (see Figure 10c), the overall good agreement between the simulations and the experiments demonstrates the capability of the model to capture the behaviour of unidirectional composites with a rather low number of material parameters. 
Furthermore, in order to validate the identified model, Figure 10d shows a comparison between the simulated and experimental data performed on a $\left[ \pm 67.5^{\circ}\right]_{4 s}$ laminate that were not used for the identification. As it can be seen, the model provides a good overall prediction for both longitudinal and transversal responses. For the transverse behaviour, both loading and unloading stages are excellently captured. With respect to the longitudinal direction, the model provides the same order of stress, but overestimates the initial slope compared to the experiment, which induces a deviation of the stress-strain predicted curve. 


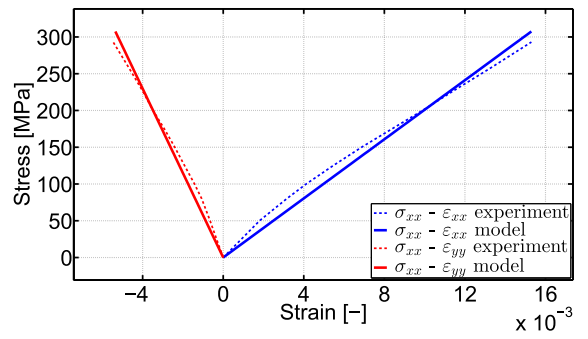

(a) Stress vs. strain on a $\left[0^{\circ}\right]_{16}$ laminate

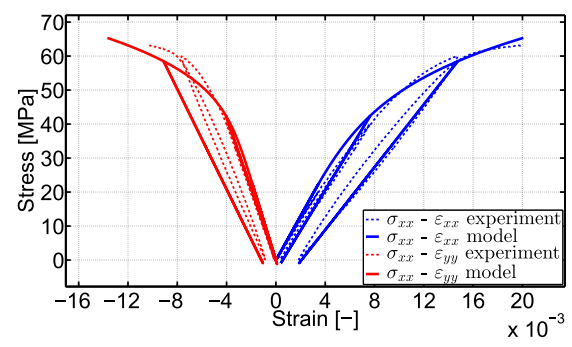

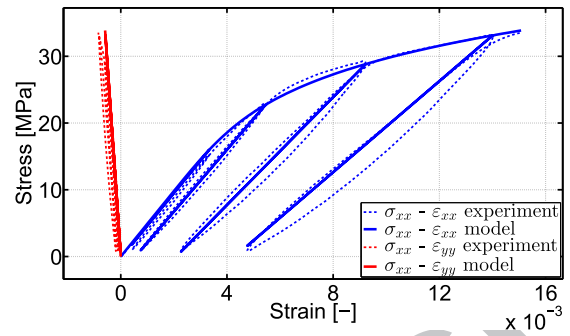

(b) Stress vs. strain on a $\left[90^{\circ}\right]_{16}$ laminate

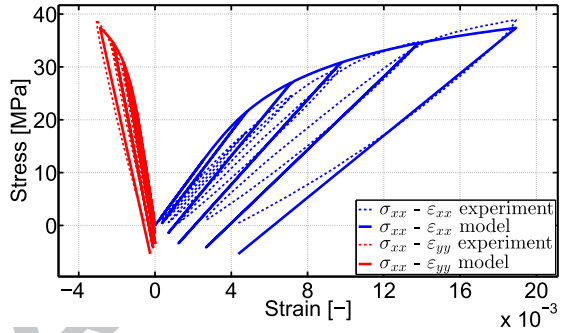

(c) Stress vs. strain on a $\left[ \pm 45^{\circ}\right]_{4 s}$ laminate

(d) Stress vs. strain on a $\left[ \pm 67.5^{\circ}\right]_{4 s}$ laminate

Figure 10: Stress $\left(\sigma_{x x}\right)$ vs. strain $\left(\varepsilon_{x x}\right.$ an $\left.\varepsilon_{y y}\right)$ for different configurations of laminate, comparison between model and experiments. The $\left[0^{\circ}\right]_{16},\left[90^{\circ}\right]_{16}$ and $\left[ \pm 45^{\circ}\right]_{4 s}$ laminates are used for the identification, while the $\left[ \pm 67.5^{\circ}\right]_{4 s}$ laminate is kept for the validation.

\section{Numerical simulations and dissipative behaviour}

To provide a better understanding regarding the dissipative behaviour as well as the interactions between transverse tension 22 and in-plane shear 12 that is accounted with the proposed constitutive model, the following simulation has been performed: The material is first loaded with a transverse normal stress $\sigma_{22}$ of $25 \mathrm{MPa}$ that is held constant for a certain period of time. While $\sigma_{22}$ is held, an in-plane shear stress $\sigma_{12}$ of $15 \mathrm{MPa}$ is applied and then released. Subsequently, the transversal stress $\sigma_{22}$ is fully unloaded (Figure 11a). 
The results of this simulation are presented in Figure 11. During the first stage $(0 \mathrm{~s}<t<40 \mathrm{~s})$, when the transverse stress $\sigma_{22}$ is applied on the material, a growth of micro-crack density is first generated and is accompanied by anelastic strain in the transverse direction (Figures 11c and 11e), both along with their related dissipations (Figure 11f). Next, the transverse stress is held and no change in the overall material response is observed, as expected, since no temporal effects are accounted for in the proposed model (no creep or relaxation). In the second stage $\left(40 \mathrm{~s}<t<80 \mathrm{~s}\right.$ ), the transverse stress $\sigma_{22}$ is still being held and an in-plane shear stress $\sigma_{12}$ is applied in addition, before being subsequently released. The micro-crack density is growing again, but at this time, anelastic strains are generated in both transverse and shear directions (Figures 11c and 11e). The material dissipates energy during the application of the shear stress and recovers a part of its stored energy when the shear stress is released (Figure 11f). Finally, in the last stage (80 $\mathrm{s}<t<$ $120 \mathrm{~s}$ ) the transverse stress is unloaded and the remaining part of the stored energy returns back to zero (Figure 11f). Indeed, as explained in Section 2.3, the model assumes that $\rho \psi$ is fully recoverable.

In terms of energy balance (Figure 11f), it can be noticed that the strain energy $W_{\varepsilon}$ is equal to the sum of the stored and dissipated energy, $\rho \psi$ and $\Phi$ respectively. Moreover, the dissipated energy is always increasing or constant, which is in accordance with the Clausius Duhem inequality (18). 


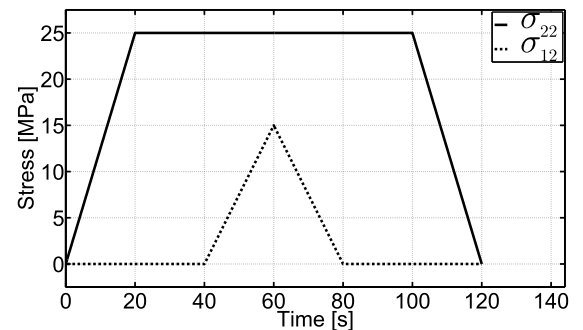

(a) Applied stress $\left(\sigma_{22}\right.$ and $\left.\sigma_{12}\right)$ vs. time

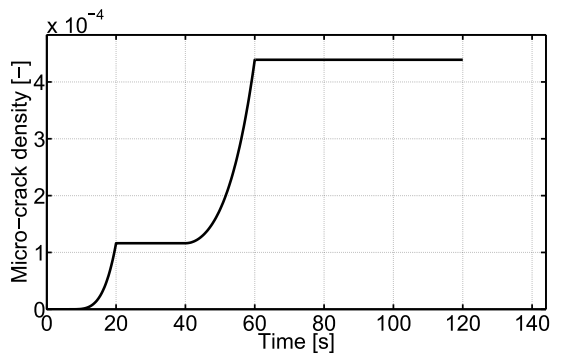

(c) Micro-crack density $\left(\gamma_{c}\right)$ vs. time

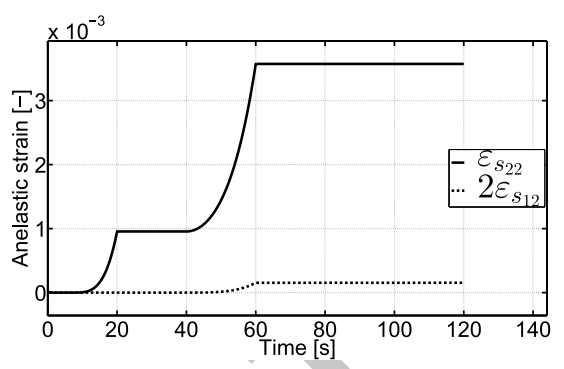

(e) Anelastic strain $\left(\varepsilon_{s_{22}}\right.$ and $\left.2 \varepsilon_{s_{12}}\right)$ vs. time

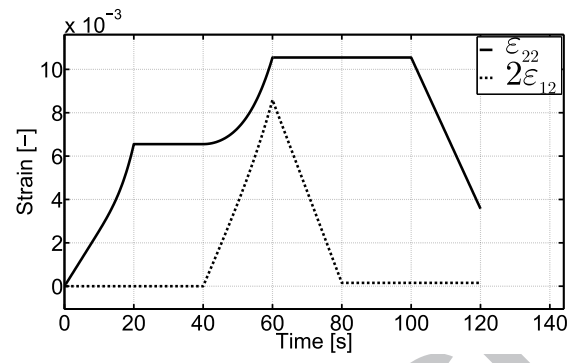

(b) Strain response $\left(\varepsilon_{22}\right.$ and $\left.2 \varepsilon_{12}\right)$ vs. time

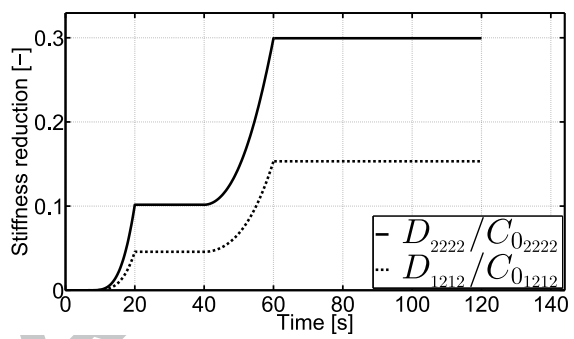

(d) Relative stiffnes reduction vs. time

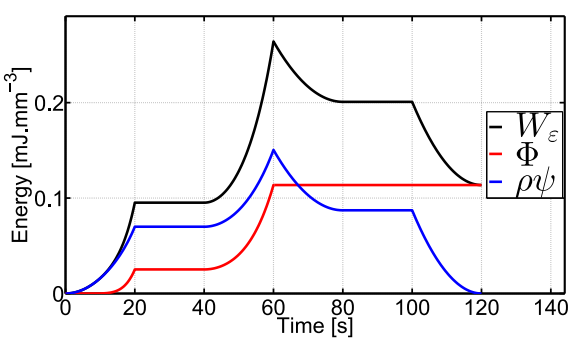

(f) Strain $\left(W_{\varepsilon}\right)$, dissipated $(\Phi)$ and stored $(\rho \psi)$ energies vs. time

Figure 11: Non-proportional bi-axial stress controlled simulation $\left(\sigma_{12}\right.$ and $\left.\sigma_{22}\right)$.

\section{Conclusions and perspectives}

A hybrid model, that predicts the mechanical behaviour of damaged unidirectional composite, has been established and implemented into a finite element code via an implicit scheme. The proposed constitutive equations are formulated in the framework of thermodynamics of irreversible processes 
with the introduction of internal state variables. Those related to damage are obtained through a micromechanical description of the the micro-crack density. The main advantages of this approach are twofold:

- The use of micromechanics allows a better description of the physical damage mechanism and its influence of the overall material response.

- Legacy CDM-based models for unidirectional composites (Le Dantec, 1989; Ladevèze and Le Dantec, 1992; Ladevèze, 1992; Allix et al., 1994; Boubakar et al., 2002, 2003; Mahboob et al., 2017) usually introduced several damage state variables and additional coupling parameters to predict the multi-axial behaviour. This may generate additional difficulties regarding their experimental identification. The present approach uses only one internal state variable and the shape of the defects to represent the overall anisotropic stiffness reduction.

The identification and the prediction capabilities has been validated on experimental data for flax-epoxy unidirectional composite. The model appears to be well adapted to describe the transverse tension and the in-plane shear responses of the composite.

As a further work, three different types of extension are proposed: i) The framework of thermodynamics gives to the model the possibility to be extended to fully coupled thermomechanical analyses. ii) The evolution equations can be enriched or/and modified in order to account for time dependent behaviours such as visco-damage (Shahsavari et al., 2016), as well as for fatigue analyses (Hochard et al., 2006; Nouri et al., 2009). Moreover, 
viscoelastic mechanisms could be eventually added through a coupled formulation (Krairi and Doghri, 2014; Krasnobrizha et al., 2016; Praud et al., 2017). iii) The proposed model will be utilized in a multi-scale modelling of woven composites, as yarn phase. Such approach can be achieved using the concept of periodic homogenization (Chatzigeorgiou et al., 2015, 2016).

\section{Acknowledgements}

This paper is part of the COPERSIM project managed by IRT Jules Verne (French Institute in Research and Technology in Advanced Manufacturing Technologies for Composite, Metallic and Hybrid Structures). The authors wish to associate the industrial and academic partners of this project; respectively Arts et Metiers ParisTech, Solvay, Plastic Omnium, PSA and Renault.

\section{Appendix A. Evaluation of the Eshelby tensor}

For a general anisotropic medium surrounding an ellipsoidal inclusion, the Eshelby tensor is given by the following surface integral, parameterized on the surface of the unit sphere (Mura, 1987). Then the components $S_{E i j k l}$ of the Eshelby tensor $\mathbb{S}_{\boldsymbol{E}}$ are given by:

$$
S_{E i j k l}=\frac{1}{8 \pi} C_{0 m n k l} \int_{-1}^{+1} d \zeta_{3} \int_{0}^{2 \pi}\left(G_{i m j n}(\bar{\zeta})+G_{j m i n}(\bar{\zeta})\right) d \omega
$$


with

$$
\begin{gathered}
G_{i j k l}(\bar{\zeta})=\frac{\bar{\zeta}_{k} \bar{\zeta}_{l} N_{i j}(\bar{\zeta})}{D(\bar{\zeta})} \\
\bar{\zeta}_{i}=\frac{\zeta_{i}}{a_{i}}, \quad \zeta_{1}=\sqrt{1-\zeta_{3}^{2}} \cos \omega, \quad \zeta_{2}=\sqrt{1-\zeta_{3}^{2}} \sin \omega, \quad \zeta_{3}=\zeta_{3} \\
D(\bar{\zeta})=\epsilon_{m n 1} K_{m 1} K_{n 2} K_{l 3}, \quad N_{i j}(\bar{\zeta})=\frac{1}{2} \epsilon_{i k l} \epsilon_{j m n} K_{k m} K_{l n}, \quad K_{i k}=C_{0 i j k l} \bar{\zeta}_{j} \bar{\zeta}_{l}
\end{gathered}
$$

$C_{0 i j k l}$ are the components of the stiffness tensor of the infinite medium $\mathbb{C}_{\mathbf{0}}$, while $a_{1}, a_{2}$ and $a_{3}$ are the three principal axes of the ellipsoid (Figure 3 ). They are expressed in a rectangular cartesian coordinate system. $\omega$ is an angular position and $\zeta_{3}$ is the longitudinal coordinate. $\epsilon_{i j k}$ is the permutation tensor expressed by:

$$
\epsilon_{i j k}= \begin{cases}0 & \text { if } i=j, i=k, j=k \\ 1 & \text { if }(i, j, k) \in\{(1,2,3),(2,3,1),(3,1,2)\} . \\ -1 & \text { if }(i, j, k) \in\{(1,3,2),(3,2,1),(2,1,3)\}\end{cases}
$$

When the infinite medium is isotropic or transversally isotropic, an analytical solution exists for (A.1). For all the other cases, no explicit formula has been developed. Thus, the components of $\mathbb{S}_{\boldsymbol{E}}$ must be evaluated numerically. As proposed by Gavazzi and Lagoudas (1990), this numerical evaluation can be performed using the following Gaussian quadrature formula:

$$
S_{E i j k l}=\frac{1}{8 \pi} \sum_{p=1}^{M} \sum_{q=1}^{N} C_{0 m n k l}\left(G_{i m j n}\left(\omega_{q}, \zeta_{3 p}\right)+G_{j m i n}\left(\omega_{q}, \zeta_{3 p}\right)\right) W_{p q}
$$

where $M$ and $N$ are the number of points used for the integration over $\zeta_{3}$ and $\omega, W_{p q}$ are the Gaussian weights. More details about the determination of the Gaussian points and weight can be found in the book by William (1989). 


\section{Appendix B. Linearisation of the constitutive equations with the "convex cutting plane" method}

Note that the "convex cutting plane" method (Ortiz and Simo, 1986; Simo and Hughes, 1998) implies a simplification regarding the linearisation of the flow equations, in which the gradients of the flows are not accounted for. Thus, the linearisation of the flow equation (26) is reduced to:

$$
\delta \varepsilon_{s}=\Lambda_{s}(\boldsymbol{\sigma}) \delta \gamma_{c}
$$

Considering this point, the linearisation of the residual (27), the criterion $H_{c}$ (19) and the stress (3) are expressed as follows:

$$
\begin{aligned}
& \delta \phi_{\gamma_{c}}=\frac{\partial \phi_{\gamma_{c}}}{\partial H_{c}} \delta H_{c}+\frac{\partial \phi_{\gamma_{c}}}{\partial \gamma_{c}} \delta \gamma_{c} \\
& =A_{\gamma_{c} H_{c}} \delta H_{c}+A_{\gamma_{c} \gamma_{c}} \delta \gamma_{c} \\
& \delta H_{c}=\frac{\partial H_{c}}{\partial \boldsymbol{\sigma}_{\mathbf{0}}}: \delta \boldsymbol{\sigma}_{\mathbf{0}} \quad \text { with from (13) } \delta \boldsymbol{\sigma}_{\mathbf{0}}=\frac{\partial \boldsymbol{\sigma}_{\mathbf{0}}}{\partial \boldsymbol{\varepsilon}} \delta \boldsymbol{\varepsilon}+\frac{\partial \boldsymbol{\sigma}_{\mathbf{0}}}{\partial \boldsymbol{\varepsilon}_{\boldsymbol{s}}} \delta \boldsymbol{\varepsilon}_{\boldsymbol{s}}+\frac{\partial \boldsymbol{\sigma}_{\mathbf{0}}}{\partial \gamma_{c}} \delta \gamma_{c} \\
& =\frac{\partial H_{c}}{\partial \boldsymbol{\sigma}_{\mathbf{0}}}:\left(\frac{\partial \boldsymbol{\sigma}_{\mathbf{0}}}{\partial \boldsymbol{\varepsilon}} \delta \boldsymbol{\varepsilon}+\frac{\partial \boldsymbol{\sigma}_{\mathbf{0}}}{\partial \boldsymbol{\varepsilon}_{\boldsymbol{s}}} \boldsymbol{\Lambda}_{\boldsymbol{s}}(\boldsymbol{\sigma}) \delta \gamma_{c}+\frac{\partial \boldsymbol{\sigma}_{\mathbf{0}}}{\partial \gamma_{c}} \delta \gamma_{c}\right) \\
& =\frac{\partial H_{c}}{\partial \boldsymbol{\sigma}_{\mathbf{0}}}: \frac{\partial \boldsymbol{\sigma}_{\mathbf{0}}}{\partial \boldsymbol{\varepsilon}}: \delta \boldsymbol{\varepsilon}+\frac{\partial H_{c}}{\partial \boldsymbol{\sigma}_{\mathbf{0}}}:\left(\frac{\partial \boldsymbol{\sigma}_{\mathbf{0}}}{\partial \boldsymbol{\varepsilon}_{\boldsymbol{s}}}: \boldsymbol{\Lambda}_{\boldsymbol{s}}(\boldsymbol{\sigma})+\frac{\partial \boldsymbol{\sigma}_{\mathbf{0}}}{\partial \gamma_{c}}\right) \delta \gamma_{c} \\
& =\boldsymbol{B}_{\boldsymbol{H}_{c} \varepsilon}: \delta \varepsilon+B_{H_{c} \gamma_{c}} \delta \gamma_{c} \\
& \delta \boldsymbol{\sigma}=\frac{\partial \boldsymbol{\sigma}}{\partial \boldsymbol{\varepsilon}}: \delta \boldsymbol{\varepsilon}+\frac{\partial \boldsymbol{\sigma}}{\partial \boldsymbol{\varepsilon}_{s}}: \delta \boldsymbol{\varepsilon}_{\boldsymbol{s}}+\frac{\partial \boldsymbol{\sigma}}{\partial \gamma_{c}} \delta \gamma_{c} \\
& =\frac{\partial \boldsymbol{\sigma}}{\partial \boldsymbol{\varepsilon}}: \delta \boldsymbol{\varepsilon}+\frac{\partial \boldsymbol{\sigma}}{\partial \varepsilon_{s}}: \boldsymbol{\Lambda}_{\boldsymbol{s}}(\boldsymbol{\sigma}) \delta \gamma_{c}+\frac{\partial \boldsymbol{\sigma}}{\partial \gamma_{c}} \delta \gamma_{c} \\
& =\frac{\partial \boldsymbol{\sigma}}{\partial \boldsymbol{\varepsilon}}: \delta \boldsymbol{\varepsilon}+\left(\frac{\partial \boldsymbol{\sigma}}{\partial \boldsymbol{\varepsilon}_{s}}: \boldsymbol{\Lambda}_{\boldsymbol{s}}(\boldsymbol{\sigma})+\frac{\partial \boldsymbol{\sigma}}{\partial \gamma_{c}}\right) \delta \gamma_{c} \\
& =\mathbb{B}_{\boldsymbol{\sigma} \varepsilon}: \delta \varepsilon+\boldsymbol{B}_{\boldsymbol{\sigma} \gamma_{c}} \delta \gamma_{c},
\end{aligned}
$$


with

$$
\begin{gathered}
A_{\gamma_{c} H_{c}}=\frac{\partial \phi_{\gamma_{c}}}{\partial H_{c}}=\frac{\partial g\left(H_{c}\right)}{\partial H_{c}} \\
A_{\gamma_{c} \gamma_{c}}=\frac{\partial \phi_{\gamma_{c}}}{\partial \gamma_{c}}=-1 \\
\boldsymbol{B}_{\boldsymbol{H}_{\boldsymbol{c}} \boldsymbol{\varepsilon}}=\frac{\partial H_{c}}{\partial \boldsymbol{\sigma}_{\mathbf{0}}}: \frac{\partial \boldsymbol{\sigma}_{\mathbf{0}}}{\partial \boldsymbol{\varepsilon}}=\left(\frac{\mathbb{H}: \boldsymbol{\sigma}_{\mathbf{0}}}{H_{c}}\right): \mathbb{C}_{\boldsymbol{0}}: \mathbb{A}_{\mathbf{0}}\left(\gamma_{c}\right) \\
B_{H_{c} \gamma_{c}}=\frac{\partial H_{c}}{\partial \boldsymbol{\sigma}_{\mathbf{0}}}:\left(\frac{\partial \boldsymbol{\sigma}_{\mathbf{0}}}{\partial \boldsymbol{\varepsilon}_{\boldsymbol{s}}} \boldsymbol{\Lambda}_{\boldsymbol{s}}(\boldsymbol{\sigma})+\frac{\partial \boldsymbol{\sigma}_{\mathbf{0}}}{\partial \gamma_{c}}\right) \\
=\left(\frac{\mathbb{H}: \boldsymbol{\sigma}_{\mathbf{0}}}{H_{c}}\right): \mathbb{C}_{\mathbf{0}}:\left(-\mathbb{A}_{\mathbf{0}}\left(\gamma_{c}\right): \boldsymbol{\Lambda}_{\boldsymbol{s}}(\boldsymbol{\sigma})+\frac{\partial \mathbb{A}_{\mathbf{0}}\left(\gamma_{c}\right)}{\partial \gamma_{c}}:\left(\boldsymbol{\varepsilon}-\boldsymbol{\varepsilon}_{\boldsymbol{s}}\right)\right) \\
\boldsymbol{B}_{\boldsymbol{\sigma} \boldsymbol{\varepsilon}}=\frac{\partial \boldsymbol{\sigma}}{\partial \boldsymbol{\varepsilon}}=\mathbb{C}_{\mathbf{0}}-\mathbb{D}_{\boldsymbol{\sigma}}\left(\gamma_{c}\right) \\
\frac{\partial \boldsymbol{\sigma}}{\partial \boldsymbol{\varepsilon}_{\boldsymbol{s}}}: \boldsymbol{\Lambda}_{\boldsymbol{s}}(\boldsymbol{\sigma})+\frac{\partial \boldsymbol{\sigma}}{\partial \gamma_{c}}=-\left[\mathbb{C}_{\mathbf{0}}-\mathbb{D}\left(\gamma_{c}\right)\right]: \boldsymbol{\Lambda}_{\boldsymbol{s}}(\boldsymbol{\sigma})-\frac{\partial \mathbb{D}\left(\gamma_{c}\right)}{\partial \gamma_{c}}:\left(\boldsymbol{\varepsilon}-\boldsymbol{\varepsilon}_{\boldsymbol{s}}\right)
\end{gathered}
$$

and

$$
\begin{gathered}
\frac{\partial \mathbb{D}\left(\gamma_{c}\right)}{\partial \gamma_{c}}=\mathbb{C}_{\mathbf{0}}: \mathbb{T}_{\boldsymbol{c}}:\left(\mathbb{A}_{\mathbf{0}}\left(\gamma_{c}\right)+\gamma_{c} \frac{\partial \mathbb{A}_{\mathbf{0}}\left(\gamma_{c}\right)}{\partial \gamma_{c}}\right), \\
\frac{\partial \mathbb{A}_{\mathbf{0}}\left(\gamma_{c}\right)}{\partial \gamma_{c}}=-\mathbb{A}_{\mathbf{0}}\left(\gamma_{c}\right):\left(\mathbb{T}_{\boldsymbol{c}}-\mathbb{I}\right): \mathbb{A}_{\mathbf{0}}\left(\gamma_{c}\right) .
\end{gathered}
$$

\section{Appendix C. Corrector}

The nullity condition of $\phi_{\gamma_{c}}$ is expressed by:

$$
\phi_{\gamma_{c}}^{(n+1)(k+1)}=\phi_{\gamma_{c}}^{(n+1)(k)}+\delta \phi_{\gamma_{c}}^{(n+1)(k)}=0
$$

where the linearisation of $\delta \phi_{\gamma_{c}}^{(n+1)(k)}$, after proper calculation (see Appendix B), takes the following form (all the quantities are taken at the increment $(n+1)(k))$ :

$$
\delta \phi_{\gamma_{c}}=A_{\gamma_{c} H_{c}} \delta H_{c}+A_{\gamma_{c} \gamma_{c}} \delta \gamma_{c}
$$


Similarly (see Appendix B), $\delta H_{c}$ is written as (recall that $\delta \boldsymbol{\varepsilon}=\mathbf{0}$ ):

$$
\delta H_{c}=B_{H_{c} \gamma_{c}} \delta \gamma_{c}
$$

By introducing (C.3) into (C.2) while considering (C.1), the unknown value of $\delta \gamma_{c}$ is computed from the known value of the residual $\phi_{\gamma_{c}}$. This gives:

$$
\delta \gamma_{c}=\frac{-\phi_{\gamma_{c}}}{K_{\gamma_{c} \gamma_{c}}} \quad \text { with } \quad K_{\gamma_{c} \gamma_{c}}=A_{\gamma_{c} H_{c}} B_{H_{c} \gamma_{c}}+A_{\gamma_{c} \gamma_{c}}
$$

where $A_{\gamma_{c} H_{c}}, A_{\gamma_{c} \gamma_{c}}$ and $B_{H_{c} \gamma_{c}}$ are provided in (B.5), (B.6) and (B.8), respectively.

\section{Appendix D. Tangent multiplier}

The condition $d \phi_{\gamma_{c}}=0$, in its linearised form (see Appendix B), is expressed by:

$$
d \phi_{\gamma_{c}}=A_{\gamma_{c} H_{c}} d H_{c}+A_{\gamma_{c} \gamma_{c}} d \gamma_{c}=0
$$

In the same manner (see Appendix B), $d H_{c}$ is written as:

$$
d H_{c}=\boldsymbol{B}_{\boldsymbol{H}_{c} \varepsilon}: d \varepsilon+B_{H_{c} \gamma_{c}} d \gamma_{c}
$$

By introducing (D.2) into (D.1), a linear relationship can be identified between $d \gamma_{c}$ and $d \varepsilon$. This gives:

$$
d \gamma_{c}=\boldsymbol{X}_{\gamma_{c} \varepsilon}: d \varepsilon \quad \text { with } \quad \boldsymbol{X}_{\gamma_{c} \varepsilon}=\frac{-A_{\gamma_{c} H_{c}} \boldsymbol{B}_{\boldsymbol{H}_{c} \varepsilon}}{K_{\gamma_{c} \gamma_{c}}}
$$

where $A_{\gamma_{c} H_{c}}, \boldsymbol{B}_{\boldsymbol{H}_{\boldsymbol{c}} \varepsilon}$ and $K_{\gamma_{c} \gamma_{c}}$ are provided in (B.5), (B.7) and (C.4), respectively.

Allix, O., Ladevèze, P., Vittecoq, E., 1994. Modelling and identification of the mechanical behaviour of composite laminates in compression. Composites science and technololgy 51, 35-42. 
Benveniste, Y., Dvorak, G.-J., Chen, T., 1991. On diagonal and elastic symmetry of the approximate effective stiffness tensor of heterogeneous media. Journal of the Mechanics and Physics of Solids 39 (7), 927-946.

Berthelot, J.-M., 1999. Matériaux composite comportement mécanique et analyse des structures, 3rd Edition. Tech \& Doc.

Boubakar, M.-L., Trivaudey, F., Perreux, D., Vang, L., 2002. A meso-macro finite element modelling of laminate structures - Part I: time-independent behaviour. Composite Structures 58, 271-286.

Boubakar, M.-L., Vang, L., Trivaudey, F., Perreux, D., 2003. A mesomacro finite element modelling of laminate structures Part II: time-dependent behaviour. Composite Structures 60, 275-305.

Chatzigeorgiou, G., Charalambakis, N., Chemisky, Y., Meraghni, F., 2016. Periodic homogenization for fully coupled thermomechanical modeling of dissipative generalized standard materials. International Journal of Plasticity $81,18-39$.

Chatzigeorgiou, G., Chemisky, Y., Meraghni, F., 2015. Computational micro to macro transitions for shape memory alloy composites using periodic homogenization. Smart Materials and Structures 24.

Couégnat, G., 2008. Approche multiéchelle du comportement mécanique de matériaux composites à renfort tissé. Ph.D. thesis, Université Bordeaux I.

Derrien, K., Fitoussi, J., Guo, G., Batiste, D., 2000. Prediction of the effective damage properties and failure properties of nonlinear anisotropic 
discontinuous reinforced composites. Computer methods in applied mechanics and engineering 185, 93-107.

Despringre, N., Chemisky, Y., Bonnay, K., Meraghni, F., 2016. Micromechanical modeling of damage and load transfer in particulate composites with partially debonded interface. Composite Structures 155, 77-88.

Dimitrijevic, B.-J., Hackl, K., 2008. A method for gradient enhancement of continuum damage models. Technische Mechanik 28 (1), 43-52.

Eshelby, J.-D., 1957. The Determination of the Elastic Field of an Ellipsoidal Inclusion, and Related Problems. Proceedings of the Royal Society of London A A (241), 376-396.

Gamstedt, E.-K., Sjögren, B.-A., 1999. Micromechanisms in tensioncompression fatigue of composite laminates containing transverse plies. Composites science and technololgy 59, 167-178.

Gavazzi, A.-C., Lagoudas, D.-C., 1990. On the numerical evaluation of Eshelby's tensor and its application to elastoplastic fibrous composites. computational Mechanics 7, 13-19.

Hashin, Z., 1980. Failure Criteria for Unidirectional Fiber Composites. Journal of Applied Mechanics 47 (2), 329-334.

Hashin, Z., Rotem, A., 1973. A Fatigue Failure Criterion for Fibre Reinforced Materials. Journal of Composite Materials 7, 448-464.

He, W., Wu, Y.-F., Xu, Y., Fu, T.-T., 2015. A thermodynamically consis- 
tent nonlocal damage model for concrete materials with unilateral effects. Computer methods in applied mechanics and engineering 297, 371-391.

Hill, R., 1948. A theory of the yielding and plastic flow of anisotropic metals. Proceedings of the Royal Society of London A 193, 281-297.

Hochard, C., Payan, J., Bordreuil, C., 2006. A progressive first ply failure model for woven ply CFRP laminates under static and fatigue loads. International Journal of Fatigue 28, 1270-1276.

Hoffman, O., 1967. The Brittle Strength of Orthotropic Materials. Journal of Composite Materials 1, 200-206.

Karafillis, A.-P., Boyce, M.-C., 1993. A general anisotropic yield criterion using bounds and transformation weighting tensor. Journal of the Mechanics and Physics of Solids 41 (12), 1959-1886.

Kiefer, B., Waffenschmidt, T., Sprave, L., Menzel, A., 2017. A gradientenhanced damage model coupled to plasticitymulti-surface formulation and algorithmic concepts. International Journal of Damage Mechanics.

Krairi, A., Doghri, I., 2014. A thermodynamically-based constitutive model, for thermoplastic polymers coupling viscoelasticity viscoplasticity and ductile damage. International Journal of Plasticity 60, 163-181.

Krasnobrizha, A., Rozycki, P., Gornet, L., Cosson, P., 2016. Hysteresis behaviour modelling of woven composite using a collaborative elastoplastic damage model with fractional derivatives. Composite Structures 158, 101111. 
Ladevèze, P., 1992. A damage computational method for composite structures. Computers \& Structures 44, 79-87.

Ladevèze, P., Le Dantec, E., 1992. Damage modelling of the elementary ply for laminated composites. Composites science and technololgy 43, 257-267.

Laws, N., Brockenbrough, J.-R., 1987. The effect of micro-crack systems on the loss of stiffness of brittle solids. International Journal of Solids and Structures 23, 1247-1268.

Laws, N., Dvorak, G.-J., Hejazi, M., 1983. Stiffness changes in unidirectional composites caused by crack systems. Mechanics of Materials 2, 123-137.

Le Dantec, E., 1989. Contribution à la modélisation du comportement mécanique des composites stratifiés. Ph.D. thesis, Université Paris VI.

Lee, H.-K., Pyo, S.-H., 2007. Micromechanics-based elastic damage modeling of particulate composites with weakened interfaces. International Journal of Solids and Structures 44, 8390-8406.

Lemaitre, J., Chaboche, J.-L., 1990. Mechanics of solid materials. Cambridge University Press.

Lemaitre, J., Desmorat, R., 2005. Engineering damage mechanics : ductile, creep, fatigue and brittle failure. Springer.

Mahboob, Z., Chemisky, Y., Meraghni, F., Bougherara, H., 2017. Mesoscale modelling of tensile response and damage evolution in natural bre reinforced laminates. Composites: Part B 119, 168-183. 
Meraghni, F., Benzeggagh, M.-L., 1995. Micromechanical modelling of matrix degradation in randomly oriented discontinuous-fibre composites. Composites science and technololgy 55, 171-186.

Meraghni, F., Blakeman, C.-J., Benzeggagh, M.-L., 1996. Effect of interfacial decohesion on stiffness reduction in a random discontinuous-fibre composite containing matrix microcracks. Composites science and technololgy 56, 541-555.

Meraghni, F., Chemisky, Y., Piotrowski, B., Echchorfi, R., Bourgeois, N., Patoor, E., 2014. Parameter identification of a thermodynamic model for superelastic shape memory alloys using analytical calculation of the sensitivity matrix. European Journal of Mechanics - A/Solids 45, 226-237.

Meraghni, F., Desrumaux, F., Benzeggagh, M.-L., 2002. Implementation of a constititive micromechanical model for damage analysis in glass mat reinforced composite structures. Composites science and technololgy 62, $2087-2097$.

Meraghni, F., Nouri, H., Bourgeois, N., Czarnota, C., Lory, P., 2011. Parameters identification of fatigue damage model for short glass fiber reinforced polyamide (PA6-GF30) using digital image correlation. Procedia Engineering $10,2110-2116$.

Mori, T., Tanaka, K., 1973. Average stress in matrix and average elastic energy of materials with misfitting inclusions. Acta Metallurgica 21, 571574. 
Mura, T., 1987. Micromechanics of defects in solids, 2nd Edition. Martinus Nijhoff publishers.

Nouri, H., Meraghni, F., Lory, P., 2009. Fatigue damage model for injectionmolded short glass fibre reinforced thermoplastics. International Journal of Fatigue 31, 934-942.

Ortiz, M., Simo, J.-C., 1986. An analysis of a new class of integration algorithms for elasto-plastic constitutive relations. International Journal for Numerical Methods in Engineering 23, 353-366.

Ottosen, N.-S., Ristinmaa, M., 2005. Failure and initial yield criteria. In: The Mechanics of Constitutive Modeling. Elsevier Science, Ch. 8, pp. 145-193.

Perreux, D., Lazuardi, D., 2001. The effect of residual stress on the non-linear behaviour of composite laminates Part II. Layer, laminate non-linear models and the effect of stress on the model parameters residual. Composites science and technololgy 61, 177-190.

Perreux, D., Oytana, C., 1993. Continuum damage mechanics for microcracked composites. Composite Engineering 3, 115-122.

Praud, F., Chatzigeorgiou, G., Bikard, J., Meraghni, F., 2017. Phenomenological multi-mechanisms constitutive modelling for thermoplastic polymers, implicit implementation and experimental validation. Mechanics of Materials 114, 9-29.

Puck, A., Schneider, W., 1969. On failure mechanisms and failure criteria of filament-wound glass-fibre/resin composites. Plastics and Polymers 37, $33-43$. 
Puck, A., Schürmann, H., 1998. Failure analysis of FRP laminates by means of physically based phenomenological models. Composites science and technololgy 58, 1045-1067.

Qi, M., Giraud, A., Colliat, J.-B., Shao, J.-F., 2016. A numerical damage model for initially anisotropic materials. International Journal of Solids and Structures 100-101, 245-256.

Qidwai, M.-A., Lagoudas, D.-C., 2000. Numerical implementation of a shape memory alloy thermomechanical constitutive model using return mapping algorithms. International Journal for Numerical Methods in Engineering 47, 1123-1168.

Qu, J., Cherkaoui, M., 2006. Fundamentals of micromechanics of solids. Wiley.

Schirmaier, F.-J., Weiland, J., Kärger, L., Henning, F., 2014. A new efficient and reliable algorithm to determine the fracture angle. Composites science and technololgy 100, 19-25.

Shahsavari, H., Naghdabadi, R., Baghani, M., Sohrabpour, S., 2016. A viscoelasticviscoplastic constitutive model considering damage evolution for time dependent materials: Application to asphalt mixes. International Journal of Damage Mechanics 25 (7), 921-942.

Simo, J.-C., Hughes, T.-J.-R., 1998. Computational inelasticity. Springer.

Tsai, S., Wu, E., 1971. A general theory of strength for anisotropic materials. Journal of Composite Materials 5, 58-80. 
Waffenschmidt, T., Polindara, C., Menzel, A., Blanco, S., 2014. A gradientenhanced large-deformation continuum damage model for fibre-reinforced materials. Computer methods in applied mechanics and engineering 268, 801-842.

Wiegand, J., Petrinic, N., Elliott, B., 2008. An algorithm for determination of the fracture angle for the three-dimensional Puck matrix failure criterion for UD composites. Composites science and technololgy 68, 2511-2517.

William, H., 1989. Numerical recipes in Fortran: The art of scientific computing. Cambridge University Press, New York.

Zaïri, F., Naït-Abdelaziz, M., Gloaguen, J.-M., Bouaziz, A., Lefebvre, J.M., 2008. Micromechanical modelling and simulation of chopped random fiber reinforced polymer composites with progressive debonding damage. International Journal of Solids and Structures 45, 5220-5236.

Zhu, Q.-Z., Shao, J.-F., Kondo, D., 2011. A micromechanics-based thermodynamic formulation of isotropic damage with unilateral and friction effects. European Journal of Mechanics - A/Solids 30, 316-325. 